\title{
Molecular tracers of radiative feedback in Orion (OMC-1) Widespread $\mathrm{CH}^{+}(J=1-0), \mathrm{CO}(10-9), \mathrm{HCN}(6-5)$,

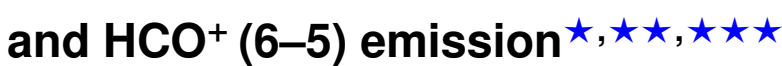

\author{
Javier R. Goicoechea ${ }^{1}$, Miriam G. Santa-Maria ${ }^{1}$, Emeric Bron ${ }^{1}$, David Teyssier ${ }^{2}$, Nuria Marcelino ${ }^{1}$, \\ José Cernicharo $^{1}$, and Sara Cuadrado ${ }^{1}$ \\ ${ }^{1}$ Instituto de Física Fundamental (CSIC), Calle Serrano 121, 28006, Madrid, Spain \\ e-mail: javier.r.goicoechea@csic.es \\ 2 Telespazio Vega UK Ltd. for ESA/ESAC, Urbanización Villafranca del Castillo, Villanueva de la Cañada, 28692 Madrid, Spain
}

Received 9 October 2018 / Accepted 30 November 2018

\begin{abstract}
Young massive stars regulate the physical conditions, ionization, and fate of their natal molecular cloud and surroundings. It is important to find tracers that quantify the stellar feedback processes that take place on different spatial scales. We present $\sim 85 \mathrm{arcmin}^{2}$ velocity-resolved maps of several submillimeter molecular lines, taken with Herschel/HIFI, toward the closest high-mass star-forming region, the Orion molecular cloud 1 core $(\mathrm{OMC}-1)$. The observed rotational lines include probes of warm and dense molecular gas that are difficult, if not impossible, to detect from ground-based telescopes: $\mathrm{CH}^{+}(J=1-0), \mathrm{CO}(J=10-9), \mathrm{HCO}^{+}(J=6-5), \mathrm{HCN}(J=6-5)$, and $\mathrm{CH}(N, J=1,3 / 2-1,1 / 2)$. These lines trace an extended but thin layer $\left(A_{\mathrm{V}} \simeq 3-6 \mathrm{mag}\right.$ or $\left.\sim 10^{16} \mathrm{~cm}\right)$ of molecular gas at high thermal pressure, $P_{\mathrm{th}}=n_{\mathrm{H}} \cdot T_{\mathrm{k}} \approx 10^{7}-10^{9} \mathrm{~cm}^{-3} \mathrm{~K}$, associated with the far-ultraviolet (FUV) irradiated surface of OMC-1. The intense FUV radiation field - emerging from massive stars in the Trapezium cluster - heats, compresses, and photoevaporates the cloud edge. It also triggers the formation of specific reactive molecules such as $\mathrm{CH}^{+}$. We find that the $\mathrm{CH}^{+}(J=1-0)$ emission spatially correlates with the flux of FUV photons impinging the cloud: $G_{0}$ from $\sim 10^{3}$ to $\sim 10^{5}$. This relationship is supported by constant-pressure photodissociation region (PDR) models in the parameter space $P_{\mathrm{th}} / G_{0} \approx\left[5 \times 10^{3}-8 \times 10^{4}\right] \mathrm{cm}^{-3} \mathrm{~K}$ where many observed PDRs seem to lie. The $\mathrm{CH}^{+}(J=1-0)$ emission also correlates with the extended infrared emission from vibrationally excited $\mathrm{H}_{2}(v \geq 1)$, and with that of [C II] $158 \mu \mathrm{m}$ and $\mathrm{CO} J=10-9$, all emerging from FUV-irradiated gas. These spatial correlations link the presence of $\mathrm{CH}^{+}$to the availability of $\mathrm{C}^{+}$ions and of FUV-pumped $\mathrm{H}_{2}(v \geq 1)$ molecules. We conclude that the parsec-scale $\mathrm{CH}^{+}$emission and narrow-line $\left(\Delta v \simeq 3 \mathrm{~km} \mathrm{~s}^{-1}\right)$ mid- $J$ CO emission arises from extended PDR gas and not from fast shocks. PDR line tracers are the smoking gun of the stellar feedback from young massive stars. The PDR cloud surface component in OMC-1, with a mass density of $120-240 M_{\odot} \mathrm{pc}^{-2}$, represents $\sim 5-10 \%$ of the total gas mass; however, it dominates the emitted line luminosity, the average CO $J=10-9$ surface luminosity in the mapped region being $\sim 35$ times brighter than that of $\mathrm{CO} J=2-1$. These results provide insights into the source of submillimeter $\mathrm{CH}^{+}$and mid- $J \mathrm{CO}$ emission from distant star-forming galaxies.
\end{abstract}

Key words. planetary nebulae: general - ISM: clouds - infrared: galaxies - galaxies: ISM

\section{Introduction}

Massive stars (>8 $\left.M_{\odot}\right)$ dominate the injection of radiative energy into the interstellar medium (ISM) through ultraviolet (UV) photons, and of mechanical energy through stellar winds, supernova explosions, and merger encounters (Beuther et al. 2007; Zinnecker \& Yorke 2007; Tan et al. 2014; Krumholz et al. 2014). Massive stars are born inside dense giant molecular cloud (GMC) cores $\left(n_{\mathrm{H}} \gtrsim 10^{5} \mathrm{~cm}^{-3}\right)$. Protostars of different masses develop inside these star-forming sites (e.g., McKee \& Ostriker 2007). Their outflows shock the ambient cloud, heating and compressing the molecular gas around them to high temperatures

\footnotetext{
${ }^{\star}$ Herschel is an ESA space observatory with science instruments provided by European-led Principal Investigator consortia and with important participation from NASA.

$\star \star$ Includes IRAM $30 \mathrm{~m}$ observations. IRAM is supported by INSU/CNRS (France), MPG (Germany), and IGN (Spain).

$\star \star \star$ All maps (FITS) are only available at the CDS via anonymous ftp to cdsarc.u-strasbg.fr $(130.79 .128 .5)$ or via http://cdsarc.u-strasbg.fr/viz-bin/qcat?J/A+A/622/A91.
}

and densities. These young protostellar systems emit, on spatial scales of $\sim 0.05 \mathrm{pc}$, high infrared (IR) luminosities, as well as vibrationally and rotationally excited $\mathrm{H}_{2}, \mathrm{CO}$, and $\mathrm{H}_{2} \mathrm{O}$ lines from shocked gas (e.g., van Dishoeck et al. 2011, and references therein). On the scales of an entire GMC, however, from several to one hundred parsec, most of the dust continuum and gas line luminosity do not arise from individual protostars but from the extended cloud component.

Once a new O-type star or cluster is formed, the energy and momentum injected by photoionization, radiation pressure, and stellar winds ionize and erode the natal molecular cloud, creating HII regions and blowing expanding bubbles (e.g., Krumholz et al. 2014; Rahner et al. 2017; Haid et al. 2018; Pabst et al. 2019). Photodisociation regions (PDRs) develop at the interfaces between the hot ionized gas and the cold molecular gas, and more generally, at any slab of neutral gas (meaning that hydrogen atoms are not ionized) illuminated by stellar far-UV (FUV) photons with energies in the range $5 \lesssim E<13.6 \mathrm{eV}$ (e.g., Hollenbach \& Tielens 1997; Goicoechea et al. 2016). 
A strong FUV radiation field ${ }^{1}\left(G_{0}>10^{3}\right)$ induces a plethora of dynamical effects (e.g., Hill \& Hollenbach 1978; Hosokawa \& Inutsuka 2006; Wareing et al. 2018; Bron et al. 2018) and chemical changes in the cloud (e.g., Hogerheijde et al. 1995; Cuadrado et al. 2015, 2016, 2017; Nagy et al. 2017; Goicoechea et al. 2017). This stellar feedback is not limited to the close vicinity of massive stars, but it can determine the gas physical conditions on scales of several parsec (e.g., Stacey et al. 1993; Herrmann et al. 1997; Goicoechea et al. 2015a) and drive the evolution of the natal cloud itself. Finding observational tracers of the radiative and mechanical feedback from massive stars is relevant not only for local studies, but also in the more general framework of star formation in galaxies. There it is not easy, at high redshifts not even possible, to disentangle where the observed line emission is coming from: embedded star-forming sites, nuclear outflows, quiescent molecular clouds, diffuse halos, etc.

The brightest FUV radiation line diagnostic is the [C II $158 \mu \mathrm{m}$ fine-structure line of ionized carbon $\mathrm{C}^{+}$ (Dalgarno \& McCray 1972). With an ionization potential of $11.3 \mathrm{eV}, \mathrm{C}^{+}$can also be abundant in both the hot ionized and the cold atomic gas (e.g., Pineda et al. 2013). Hence, it is not always trivial to establish the origin of the [C II] $158 \mu \mathrm{m}$ line and exploit its full diagnostic power (e.g., Pabst et al. 2017). In consequence, it is important to have diverse observational probes of stellar feedback processes.

Reactive ions such as $\mathrm{CH}^{+}, \mathrm{OH}^{+}$, or $\mathrm{SH}^{+}$are among the first molecules to form in initially atomic gas. That is, $x(\mathrm{H}$ atoms $)>x\left(\mathrm{H}_{2}\right.$ molecules $)$, where $x$ refers to the species abundance with respect to $\mathrm{H}$ nuclei (for a review see Gerin et al. 2016). In FUV-irradiated neutral gas, carbon is mainly in the form of $\mathrm{C}^{+}$(Goldsmith et al. 2012; Gerin et al. 2015). The formation rate of $\mathrm{CH}^{+}$depends on the flux of FUV photons, the gas temperature, and the abundance of vibrationally excited $\mathrm{H}_{2}(v \geq 1)$; either collisionally excited or FUV-pumped (Sternberg \& Dalgarno 1995; Agúndez et al. 2010). The main gas-phase reaction producing detectable quantities of $\mathrm{CH}^{+}$is

$\mathrm{C}^{+}+\mathrm{H}_{2}(v) \rightarrow \mathrm{CH}^{+}+\mathrm{H}$

where $v$ refers to the specific vibrational level of $\mathrm{H}_{2}$. This reaction has a very high endothermicity, $\Delta E / k \simeq 4300 \mathrm{~K}$, if $v=0$. Thus, one would expect negligible $\mathrm{CH}^{+}$abundances in molecular clouds (where $T_{\mathrm{k}} \ll \Delta E / k$ ). Reaction (1), however, becomes exothermic and fast for $v \geq 1$ (Hierl et al. 1997; Zanchet et al. 2013), with the first $\mathrm{H}_{2}$ vibrational level lying at $E_{v=1} / k \simeq 5987 \mathrm{~K}$. Hence, compared to other molecules, $\mathrm{CH}^{+}$is expected to be abundant only in high-temperature FUV-irradiated gas (e.g., Black 1998; Gerin et al. 2016).

Once formed, $\mathrm{CH}^{+}$is a very reactive ion; the timescale for its destruction in reactions with $\mathrm{H}_{2}$ molecules or $\mathrm{H}$ atoms is comparable to, or shorter than, that for non-reactive collisions (elastic or inelastic). In dense gas, $n_{\mathrm{H}} \gtrsim 10^{5} \mathrm{~cm}^{-3}$, the lifetime of $\mathrm{CH}^{+}$is so short, a few hours, that the molecule may form and be destroyed without experiencing many non-reactive collisions with other species (Black 1998; Nagy et al. 2013; Godard \& Cernicharo 2013; Goicoechea et al. 2017; Faure et al. 2017).

$\mathrm{CH}^{+}$was one of the first interstellar molecules detected in the 1930s (Douglas \& Herzberg 1941). Due to the high endothermicity of reaction (1) and limited $\mathrm{H}_{2}(v \geq 1)$ column densities in low-density gas, explaining the presence of $\mathrm{CH}^{+}$

\footnotetext{
$1 G_{0}=1$ is equal to $1.6 \times 10^{-3} \mathrm{erg} \mathrm{cm}^{-2} \mathrm{~s}^{-1}$, the FUV flux (integrated from $\sim 912$ to $2400 \AA$ ) in the solar neighborhood (Habing 1968).
}

in diffuse clouds (typically with $G_{0} \approx 1, n_{\mathrm{H}} \lesssim 100 \mathrm{~cm}^{-3}$, and $T_{k} \lesssim 100 \mathrm{~K}$ ) has been a long standing problem in astrochemistry. At such low densities, the $\mathrm{CH}^{+} J=1-0$ line can only be detected in absorption against bright submillimeter (submm) continuum background sources (Falgarone et al. 2010a; Godard et al. 2012). Low-velocity shocks (Elitzur \& Watson 1978; Draine \& Katz 1986; Pineau des Forets et al. 1986) and, in particular, intermittent turbulence dissipation in a magnetized medium (Falgarone et al. 1995; Joulain et al. 1998; Godard et al. $2009,2014)$ are the most promising scenarios to explain the presence of $\mathrm{CH}^{+}$in diffuse gas. These theories are based in a local enhancement of the gas heating that raises the temperature to $T_{\mathrm{k}} \gtrsim 1000 \mathrm{~K}$ in shear structures or vortices of only a few hundred AU. At these high temperatures, and enhanced velocity drift between ions and neutrals, reaction (1) becomes efficient. Other, perhaps less succesful, scenarios include the turbulent mixing between the warm and the cold neutral phases of the ISM (Lesaffre et al. 2007) and the presence of extended warm $\mathrm{H}_{2}$ (Valdivia et al. 2017).

The detection of far-IR (FIR) $\mathrm{CH}^{+}$rotational line emission from strongly FUV-irradiated dense PDRs $\left(G_{0}>10^{3}\right.$, $n_{\mathrm{H}} \gtrsim 10^{5} \mathrm{~cm}^{-3}$; Cernicharo et al. 1997; Naylor et al. 2010; Nagy et al. 2013; Pilleri et al. 2014; Joblin et al. 2018) suggested that the more intense FUV field and much higher densities compared to diffuse clouds enhances the abundance of $\mathrm{H}_{2}(v \geq 1)$ and also the $\mathrm{CH}^{+}$production. Indeed, models using updated stateto-state chemical rates for reaction(1) emphasized the role of FUV-pumped $\mathrm{H}_{2}(v \geq 1)$ to explain the presence of $\mathrm{CH}^{+}$in bright and dense PDRs (Agúndez et al. 2010; Nagy et al. 2013; Faure et al. 2017). Later observations showed that $\mathrm{CH}^{+}$can be relatively extended near young massive stars (Morris et al. 2016). In addition, Herschel also detected $\mathrm{CH}^{+}$broad-line emission from high- and low-mass protostars (Falgarone et al. 2010b; Benz et al. 2010, 2016). The preferred interpretation is that $\mathrm{CH}^{+}$forms in irradiated magnetized shocks: the FUV-irradiated walls of a protostellar outflow. Last but not least, $\mathrm{CH}^{+}(J=1-0)$ emission has been recently detected by ALMA in star-forming galaxies at redshift of about two (Falgarone et al. 2017).

In summary, $\mathrm{CH}^{+}$has been historically considered a unique, sometimes exotic, tracer of harsh conditions (Black 1998). Despite many theoretical efforts, there have not been any observational study on how the extended $\mathrm{CH}^{+}$emission relates with the gas physical conditions and with the flux of FUV photons. In this paper we present the detection of large-scale $\mathrm{CH}^{+}(J=1-0)$ emission toward Orion molecular cloud 1 (OMC-1), complemented with velocity-resolved maps of other key diagnostics of the warm molecular gas that help to understand the role of stellar radiation at large spatial scales. The paper is organized as follows. In Sect. 2 we introduce the region and describe the observations. In Sect. 3 we present the main observational results. In Sect. 4 we analyse the maps, focusing on the $\mathrm{CH}^{+}$ emission, and in Sect. 5 we model and discuss the role of FUV radiation.

\section{Observations and data reduction}

\subsection{OMC-1 in Orion $A$}

OMC-1, in the Orion A complex, lies behind the iconic Orion Nebula (M42) and the massive stars $\theta^{1}$ Ori of the Trapezium cluster (with spectral types from O7V to B3V; e.g., Simón-Díaz et al. 2006) roughly at the center of the Orion nebula cluster (ONC) (e.g., Zuckerman 1973; Genzel \& Stutzki 1989; 


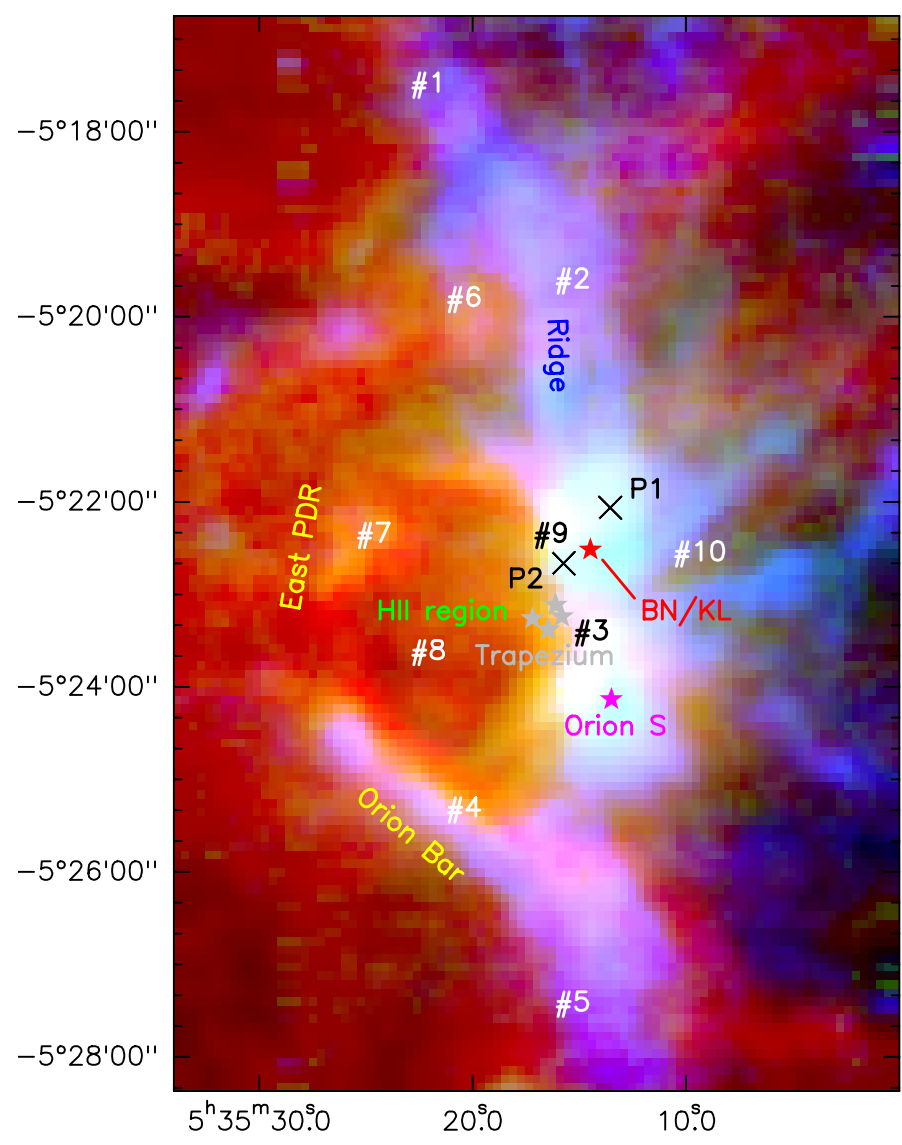

Fig. 1. RGB multitracer view of OMC-1 at $\sim 12^{\prime \prime}$ resolution. Red: [C II] $158 \mu \mathrm{m}$ emission from FUV-illuminated surface of the molecular cloud (Goicoechea et al. 2015a). Green: $\mathrm{HCO}^{+}(J=3-2)$ from warm and dense gas. Blue: $\mathrm{C}^{18} \mathrm{O}(J=2-1)$ from colder and more FUV-shielded gas. Main regions in OMC-1 are labeled and representative positions discussed in the text are indicated with numbers.

O'Dell 2001). At a distance ${ }^{2}$ of $\sim 414$ pc (Menten et al. 2007), OMC-1 is the closest region of on-going intermediate- and high-mass star formation. The two major star-forming sites are the Becklin-Neugebauer/Kleinmann-Low region (BN/KL; Becklin \& Neugebauer 1967) and Orion South (Orion S; Fig. 1). $\mathrm{BN} / \mathrm{KL}$ hosts a high-velocity wide-angle outflow that contains the two brightest IR $\mathrm{H}_{2}$ emission peaks in the sky: Peak 1 (P1) and Peak 2 (P2), produced by hot shocked gas (marked with crosses in Fig. 1; see Kwan \& Scoville 1976; Beckwith et al. 1978; González-Alfonso et al. 2002; Goicoechea et al. 2015b). Both BN/KL and Orion S show chemically rich spectra (e.g., Blake et al. 1987; Tercero et al. 2010; Tahani et al. 2016). OMC-1 is directly exposed to the strong FUV radiation emitted by young hot stars in the Trapezium, and in particular dominated by $\operatorname{star} \theta^{1}$ Ori $\mathrm{C} 1\left(M_{\star} \simeq 33.5 M_{\odot}\right.$; e.g., GRAVITY Collaboration et al. 2018) located at $\sim 0.3 \mathrm{pc}$ in front of the molecular cloud. The FUV-irradiated surface of OMC-1, to a first approximation a large-scale face-on PDR, copiously emits in FIR [C II] $158 \mu \mathrm{m}$ and [O I] $63 \mu \mathrm{m}$ fine-structure gas-cooling lines (Stacey et al. 1993; Herrmann et al. 1997; Goicoechea et al. 2015a).

Velocity-resolved maps over tens of square arcmin are needed to constrain the kinematics and physical properties

\footnotetext{
2 Recent estimations, from Gaia DR2, suggest that the distance to ONC is lower, $386 \pm 3$ pc (e.g., Großschedl et al. 2018, and references therein). Here we still adopt $\sim 414$ pc to be consistent with the luminosities computed in our previous works (e.g., Goicoechea et al. 2015a,b).
}

of the extended, non-star-forming gas in GMCs. A number of studies have provided such molecular line maps toward OMC-1. However, most of them focus on the lowest-energy rotational lines that typically trace cold molecular gas (e.g., Bally et al. 1987; Goldsmith et al. 1997; Ungerechts et al. 1997; Berné et al. 2014; Kauffmann et al. 2017). Indeed, the warm molecular gas, $T_{k} \approx 100 \mathrm{~K}$, predominantly emits in higher frequency lines. Schmid-Burgk et al. (1989) presented a pioneering velocity-resolved CO $J=7-6$ line map ( 48 $\left.\operatorname{arcmin}^{2}\right)$ of OMC-1 taken with $K A O$ at $\sim 100^{\prime \prime}$ angular resolution. Improved angular resolution maps were later taken from ground-based telescopes in CO $J=7-6\left(\sim 8 \operatorname{arcmin}^{2}\right.$; Wilson et al. 2001) and up to CO $J=8-7\left(\sim 30 \operatorname{arcmin}^{2}\right.$; Peng et al. 2012). The narrow CO line profiles $\left(\Delta v \lesssim 4 \mathrm{~km} \mathrm{~s}^{-1}\right)$ observed toward many positions suggested the widespread nature of warm (but not shocked) molecular gas. In addition, the spatial distribution of the extended $\mathrm{CN}$ emission implied an active photochemistry at the interface between the H II regions M42 and M43 and the molecular cloud (Rodríguez-Franco et al. 1998, 2001).

\subsection{Herschel/HIFI and PACS maps}

In this work we present new submm line maps of OMC-1 taken with the heterodyne instrument HIFI (de Graauw et al. 2010) on board Herschel space telescope (Pilbratt et al. 2010). The maps have a size of $\sim 85 \operatorname{arcmin}^{2}(\sim 0.9 \mathrm{pc} \times 1.4 \mathrm{pc})$. They belong to program OT1_jgoicoec_4. A [C II] $158 \mu \mathrm{m}$ map from this program was first presented in Goicoechea et al. (2015a). Morris et al. (2016) previosulsy presented smaller $\left(\sim 12 \operatorname{arcmin}^{2}\right)$ maps around Orion BN/KL based on observations performed during the regular instrument calibration activities. At the observed frequencies, HIFI employed Superconductor-Insulator-Superconductor (SIS) mixers in two orthogonal polarizations. We used the Wide Band Acousto-Optical Spectrometer providing a spectral resolution of $1.1 \mathrm{MHz}$ and a bandwidth of $4 \mathrm{GHz}$. We obtained on-the-fly (OTF) maps with half-beam sampling, and angular resolutions (half-power beam-widths, HPBW) ranging from $43^{\prime \prime}$ in Band 1 to $20^{\prime \prime}$ in Band 5. A reference-OFF position at $9^{\prime}$ was observed. Total map integration times were $\sim 1.4 \mathrm{~h}$ at $\sim 535 \mathrm{GHz}$, $\sim 2.2 \mathrm{~h}$ at $\sim 835 \mathrm{GHz}$, and $\sim 4.6 \mathrm{~h}$ at $\sim 1152 \mathrm{GHz}$. We used the main beam temperature scale $\left(T_{\mathrm{mb}}\right.$ in $\left.\mathrm{K}\right)$ as opposed to $T_{\mathrm{A}}^{*}$. For semi-extended emission sources, not infinite but larger than HIFI's HPBW, of uniform brightness temperature $\left(T_{\mathrm{b}}\right), T_{\mathrm{mb}}$ is the most appropriate intensity scale $\left(T_{\mathrm{mb}} \simeq T_{\mathrm{b}}\right)$. The achieved rms noise was typically $50 \mathrm{mK}$ at $\sim 531 \mathrm{GHz}, \sim 100 \mathrm{mK}$ at $\sim 835$ and $\sim 1152 \mathrm{GHz}$, and $\sim 800 \mathrm{mK}$ per channel.

As part of a related Herschel program (KPGT_ebergin_l), we used the PACS spectrometer (Poglitsch et al. 2010) to map a $\sim 2^{\prime} \times 2^{\prime}$ region around Orion BN/KL and the Trapezium cluster area. These $\sim 12^{\prime \prime}$ angular resolution observations cover the shorter $\lambda=70-94 \mu \mathrm{m}$ and $108-190 \mu \mathrm{m}$ wavelength ranges where $\mathrm{CH}^{+}$rotationally excited lines appear. PACS map data calibration and line flux extraction are described in Goicoechea et al. (2015b). Here we present the detection of bright $\mathrm{CH}^{+} J=3-2$, 4-3, and 5-4 lines toward a position close to the Trapezium cluster: position \#3 (see Figs. 1 and 2).

\subsection{IRAM $30 \mathrm{~m}$ maps}

We complemented our study with the analysis of unpublished $\mathrm{C}^{18} \mathrm{O}(J=2-1)$ and $\mathrm{HCO}^{+}(J=3-2)$ line maps, obtained by us with the multi-beam receiver HERA at the IRAM 30m telescope (Pico Veleta, Spain). The HPBW ranges from $\sim 9^{\prime \prime}$ to $11^{\prime \prime}$. We used the versatile spectrometer VESPA as a backend providing $320 \mathrm{kHz}$ of spectral resolution $\left(0.4 \mathrm{~km} \mathrm{~s}^{-1}\right)$. These 

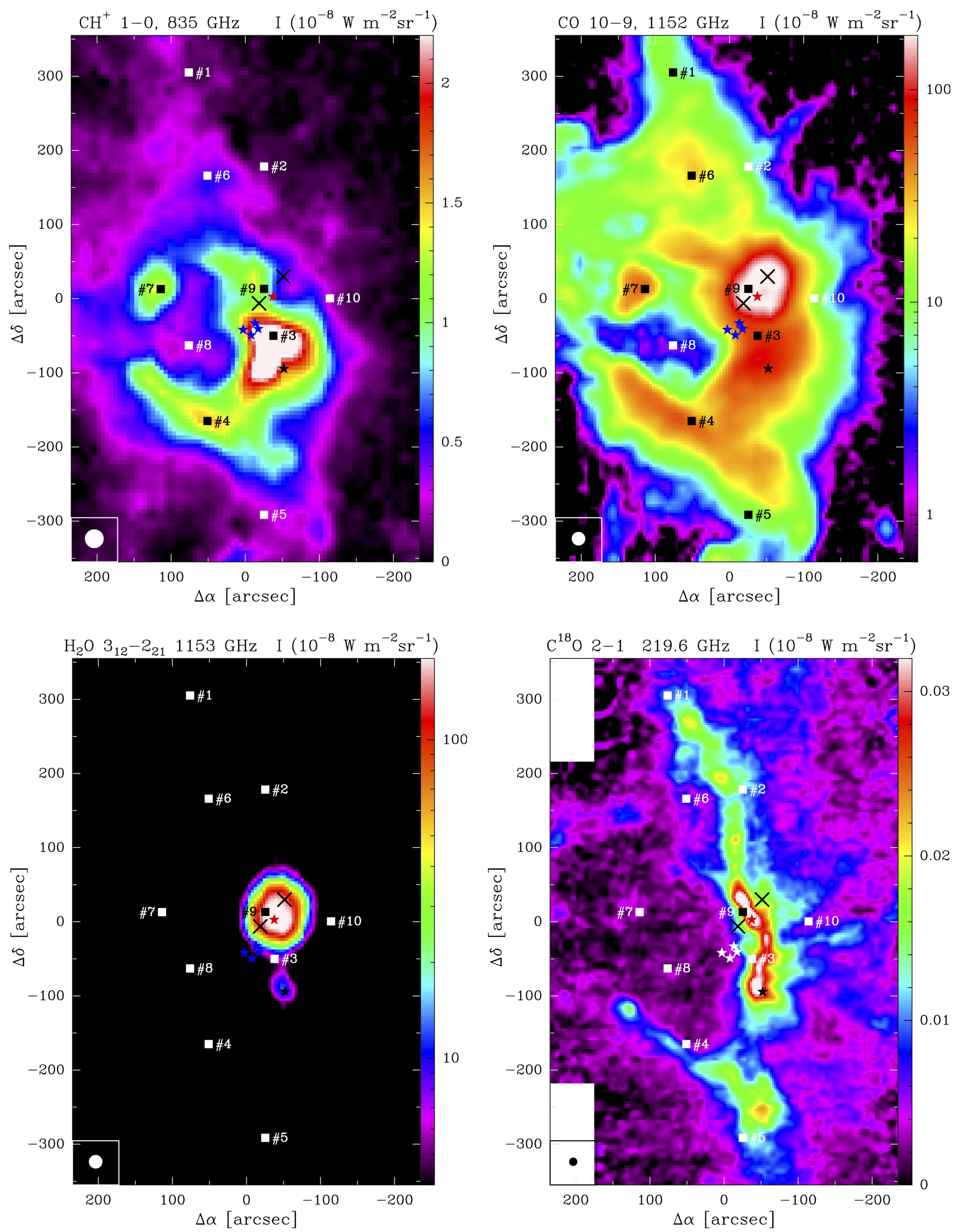

Fig. 2. Herschel/HIFI and IRAM 30m maps of different molecular emission lines toward OMC-1, all showing very different spatial distributions: $\mathrm{CH}^{+} J=1-0$ (strongly FUV-irradiated gas), $\mathrm{CO} J=10-9$ (extended warm gas), $\mathrm{H}_{2} \mathrm{O} 3_{12}-2_{21}$ (hot shocked gas), and $\mathrm{C}^{18} \mathrm{O} J=2-1$ (colder and more FUV-shielded molecular gas). The color scale shows the integrated line intensity in units of $\mathrm{W} \mathrm{m}^{-2} \mathrm{sr}^{-1}$. The native angular resolution of each observation, the HPBW, is plotted in the bottom-left corner. Representative positions discussed in the text are indicated with numbers. 
Table 1. Transition frequencies, upper energy levels, Einstein coefficients, and critical densities discussed in this work (sorted by frequencies).

\begin{tabular}{|c|c|c|c|c|c|c|c|c|}
\hline Species & Transition & $\begin{array}{r}\text { Frequency } \\
(\mathrm{GHz})\end{array}$ & $\begin{array}{r}E_{\mathrm{u}} / k \\
(\mathrm{~K})\end{array}$ & $\begin{array}{c}A_{\mathrm{ul}} \\
\left(\mathrm{s}^{-1}\right) \\
\end{array}$ & $\begin{array}{r}n_{\mathrm{cr}}^{a} \\
\left(\mathrm{~cm}^{-3}\right) \\
\end{array}$ & $\begin{array}{r}\text { Telescope } \\
\text { /Instrument }\end{array}$ & $\begin{array}{r}\text { HPBW } \\
\left({ }^{\prime \prime}\right) \\
\end{array}$ & $\begin{array}{r}\text { Herschel } \\
\text { ObsID }\end{array}$ \\
\hline $\mathrm{CH}^{+}$ & $J=5-4$ & 4155.8719 & 599.6 & 1.05 & $8 \times 10^{10}$ & Herschel/PACS & $\sim 12$ & $342218571 / 2$ \\
\hline $\mathrm{CH}^{+}$ & $J=4-3$ & 3330.6297 & 400.1 & 0.53 & $2 \times 10^{10}$ & /PACS & $\sim 12$ & $342218571 / 2$ \\
\hline $\mathrm{CH}^{+}$ & $J=3-2$ & 2501.4404 & 240.3 & 0.22 & $5 \times 10^{9}$ & /PACS & $\sim 12$ & $342218571 / 2$ \\
\hline $\mathrm{C}^{+\dagger}$ & ${ }^{2} P_{3 / 2}{ }^{2} P_{1 / 2}$ & 1900.5369 & 91.3 & $2.3 \times 10^{-6}$ & $4 \times 10^{3}$ & Herschel/HIFI & 12.0 & $1342250412 / 4 / 5$ \\
\hline$o-\mathrm{H}_{2} \mathrm{O}$ & $3_{12}-221$ & 1153.1268 & 215.2 & $2.7 \times 10^{-3}$ & $\sim 6 \times 10^{7}$ & /HIFI & 19.8 & 1342250465 \\
\hline${ }^{12} \mathrm{CO}$ & $J=10-9$ & 1151.9854 & 304.2 & $1.0 \times 10^{-4}$ & $1 \times 10^{6}$ & /HIFI & 19.8 & 1342250465 \\
\hline $\mathrm{HCN}$ & $J=13-12$ & 1151.4491 & 387.0 & $7.6 \times 10^{-2}$ & $6 \times 10^{9}$ & /HIFI & 19.8 & 1342250465 \\
\hline $\mathrm{CH}^{+}$ & $J=1-0$ & 835.1375 & 40.1 & $6.2 \times 10^{-3}$ & $1 \times 10^{8}$ & /HIFI & 27.3 & 1342250217 \\
\hline $\mathrm{HCO}^{+}$ & $J=6-5$ & 535.0616 & 89.9 & $1.2 \times 10^{-2}$ & $3 \times 10^{7}$ & /HIFI & 42.6 & 1342244307 \\
\hline $\mathrm{CH}$ & $N, J=1,3 / 2-1,1 / 2$ & 532.7933 & 25.7 & $4.1 \times 10^{-4}$ & $\sim 3 \times 10^{8}$ & /HIFI & 42.8 & 1342244307 \\
\hline $\mathrm{HCN}$ & $J=6-5$ & 531.7164 & 89.3 & $7.2 \times 10^{-3}$ & $7 \times 10^{8}$ & /HIFI & 42.8 & 1342244307 \\
\hline$A-\mathrm{CH}_{3} \mathrm{OH}$ & $11_{0,11}-10_{0,10}$ & 531.3193 & 153.1 & $6.8 \times 10^{-4}$ & $\sim 5 \times 10^{6}$ & /HIFI & 42.9 & 1342244307 \\
\hline $\mathrm{HCO}^{+}$ & $J=3-2$ & 267.558 & 25.7 & $1.4 \times 10^{-3}$ & $4 \times 10^{6}$ & IRAM 30m/HERA & 9.2 & \\
\hline${ }^{12} \mathrm{CO}^{\ddagger}$ & $J=2-1$ & 230.538 & 16.6 & $6.9 \times 10^{-7}$ & $1 \times 10^{4}$ & /HERA & 10.7 & \\
\hline${ }^{13} \mathrm{CO}^{\ddagger}$ & $J=2-1$ & 220.399 & 15.9 & $6.1 \times 10^{-7}$ & $1 \times 10^{4}$ & /HERA & 11.2 & \\
\hline $\mathrm{C}^{18} \mathrm{O}$ & $J=2-1$ & 219.560 & 15.8 & $6.0 \times 10^{-7}$ & $1 \times 10^{4}$ & /HERA & 11.2 & \\
\hline
\end{tabular}

Notes. ${ }^{(a)}$ Critical density, $A_{\mathrm{ul}} / \gamma_{\mathrm{ul}}\left(T_{\mathrm{k}}\right)$, in collisions with $\mathrm{H}_{2}$ at $100 \mathrm{~K}$.

References. ${ }^{\dagger}$ Published in Goicoechea et al. (2015a). ${ }^{\dagger}$ Published in Berné et al. (2014).

maps belong to a project to map a much larger area of Orion A in $\mathrm{CO}$ isotopologues. The data reduction is described in Berné et al. (2014). The achieved rms noise is $\simeq 0.2 \mathrm{~K}\left(\mathrm{C}^{18} \mathrm{O} J=2-1\right)$ and $\simeq 1 \mathrm{~K}\left(\mathrm{HCO}^{+} J=3-2\right)$ per $0.4 \mathrm{~km} \mathrm{~s}^{-1}$ channel.

Herschel and IRAM $30 \mathrm{~m}$ data cubes were processed with GILDAS. Figure 2 and also Figs. B.1 and B.2 show the resulting emission maps at their native angular resolutions. Offsets in arcsec are given with respect to the maps center at $\alpha_{2000}: 5^{\mathrm{h}} 35^{\mathrm{m}} 17.0^{\mathrm{s}}, \delta_{2000}:-5^{\circ} 22^{\prime} 33.7^{\prime \prime}$. Table 1 summarizes the main spectroscopic parameters of the observed lines. To determine the total luminosity emitted by each line, we converted the integrated line intensity maps from $\mathrm{K} \mathrm{km} \mathrm{s}^{-1}$ units to line surface brightness in $\mathrm{W} \mathrm{m}^{-2} \mathrm{sr}^{-1}$. The conversion ${ }^{3}$ scales with the cube of the line frequency. Hence, it greatly affects the comparison of FIR, submm and mm lines. Finally, to match the $\mathrm{CH}^{+} J=1-0$ $\left(\mathrm{HCO}^{+} J=6-5\right)$ angular resolution and carry out a combined analysis (e.g., line ratio maps), we also convolved the maps to an uniform resolution of $27^{\prime \prime}\left(43^{\prime \prime}\right)$.

\section{Results}

\subsection{Global spatial distribution of different line tracers}

Figure 1 shows a composite image of the integrated line emission in: [C II] $158 \mu \mathrm{m}$ (red) from the FUV-irradiated surfaces of $\mathrm{OMC}-1 ; \mathrm{HCO}^{+} J=3-2$ (green) from warm and dense molecular gas; and $\mathrm{C}^{18} \mathrm{O} J=2-1$ (blue) from colder and more FUV-shielded gas, mostly tracing the cloud interior (e.g., Bally et al. 1987; Hacar et al. 2017, 2018). The strong flux of FUV photons from the Trapezium illuminates the surface of the molecular cloud and creates bright and dense PDRs. The most noticeable edge-on $\mathrm{HII} / \mathrm{H} / \mathrm{H}_{2}$ interfaces are the Orion Bar and the East PDRs, but the entire illuminated skin of the cloud, bright in [C II] $158 \mu \mathrm{m}$ emission (Fig. B.3) can be seen as a face-on PDR (e.g., Stacey et al. 1993; Goicoechea et al. 2015a).

Figure 2 shows maps of the $\mathrm{CH}^{+} \quad J=1-0$, CO $J=10-9$, $\mathrm{H}_{2} \mathrm{O} 3_{12}-2_{21}$, and $\mathrm{C}^{18} \mathrm{O} J=2-1$ lines. The four emission lines show remarkably different spatial distributions, emphasizing the distinctive diagnostic power of different molecular species and lines. Although much less abundant than $\mathrm{C}^{+}$, the spatial distribution of $\mathrm{CH}^{+}(J=1-0)$ is similar to that of [C II] $158 \mu \mathrm{m}$ (Figs. 3 and B.3). In particular, the $\mathrm{CH}^{+} J=1-0$ intensity peaks appear very close to those of [C II] $158 \mu \mathrm{m}$. Hence, $\mathrm{CH}^{+}$also traces FUV-irradiated gas at the surface of the molecular cloud. However, while the brightest regions of [C II] $158 \mu \mathrm{m}$ emission show uniform intensities, the $\mathrm{CH}^{+} J=1-0$ line is brighter closer to the Trapezium, where the stellar FUV flux is stronger. Of all the observed species, only the $\mathrm{CH}$ emission shows a similar spatial distribution to that of $\mathrm{CH}^{+}$(Fig. B.1). This is a consequence of their very related chemistry in PDR gas (see Morris et al. 2016).

Despite the high excitation requirements of the $\mathrm{CO}$ rotational level $J=10\left(E_{\mathrm{u}} / k=304 \mathrm{~K}\right)$, thus only populated at warm gas temperatures, the $\mathrm{CO} J=10-9$ line emission is very extended. Indeed, it is more spatially extended than that of $\mathrm{C}^{18} \mathrm{O}(J=2-1)$ (Fig. 2). Unlike $\mathrm{CH}^{+}$and $\mathrm{CH}$, the $\mathrm{CO} J=10-9$ emission peaks toward BN/KL. However, even if it locally peaks toward shocked gas in outflows, most of the line luminosity arises from the extended cloud component. In addition, outside the BK/KL region, the spatial distribution of the $\mathrm{CO} J=10-9$ emission approximately follows that of $\mathrm{CH}^{+}$and [C II] $158 \mu \mathrm{m}$ (see Fig. 3). Together with the narrow CO linewidths, $\Delta v \simeq 3 \mathrm{~km} \mathrm{~s}^{-1}$, this suggests that most of the $\mathrm{CO} J=10-9$ emission also arises from the warm FUV-irradiated surface of OMC-1.

Among the species studied in this work, the detection of broad $\left(\Delta v>30 \mathrm{~km} \mathrm{~s}^{-1}\right)$ line-wing $\mathrm{H}_{2} \mathrm{O}\left(3_{12}-2_{21}\right)$ emission implies the presence of shocked gas activity (e.g., van Dishoeck et al. 2011, 2013). Indeed, we only detect $\mathrm{H}_{2} \mathrm{O}$ and $\mathrm{CH}_{3} \mathrm{OH}$ rotationally excited lines toward Orion $\mathrm{S}$ and BN/KL (Figs. 2 and B.1). Both species are abundant in the ice mantles that coat grains in cold dark clouds (e.g., Gibb et al. 2004). After the onset of protostellar outflows, high-velocity shocks sputter these grain mantles and heat the gas to high temperatures. Both effects enhance the abundance of gas-phase $\mathrm{H}_{2} \mathrm{O}$ and $\mathrm{CH}_{3} \mathrm{OH}$ (e.g., Draine 1995; Jiménez-Serra et al. 2008). In OMC-1, the 

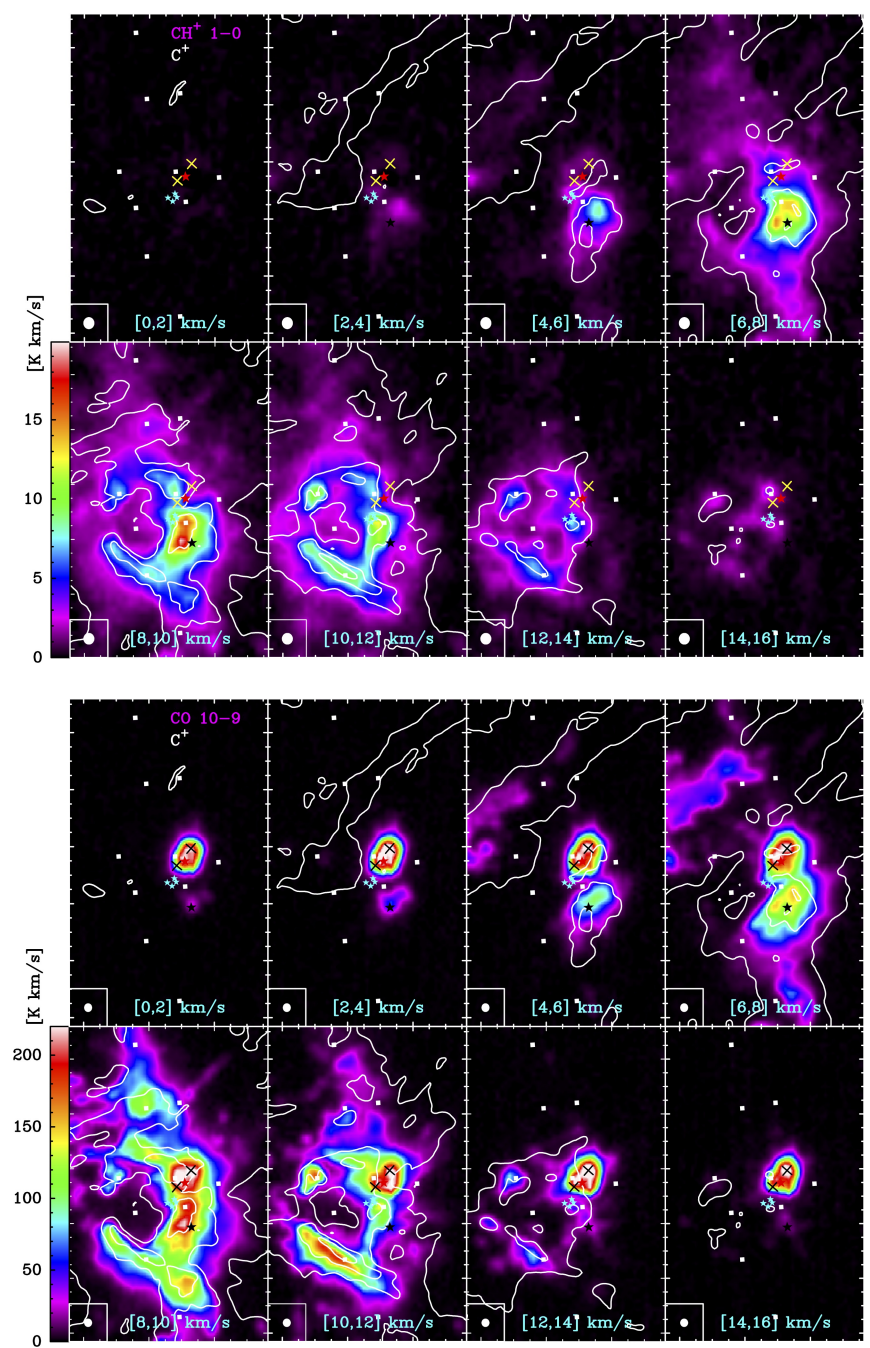

Fig. 3. $\mathrm{CH}^{+} J=1-0$ (top panel) and CO $J=10-9$ (bottom panel) velocity channel maps from $v_{\mathrm{LSR}}=0$ to $16 \mathrm{~km} \mathrm{~s}^{-1}$ in steps of $2 \mathrm{~km} \mathrm{~s}^{-1}$. White contours show the [C II] $158 \mu \mathrm{m}$ line emission from 50 to $500 \mathrm{~K} \mathrm{~km} \mathrm{~s}^{-1}$ in steps of $150 \mathrm{~K} \mathrm{~km} \mathrm{~s}^{-1}$ (Goicoechea et al. 2015a).

low- and high-velocity outflows from BN/KL plunge into the ambient molecular cloud (Genzel \& Stutzki 1989) producing hot (from $T_{\mathrm{k}} \simeq 200-2000 \mathrm{~K}$ ) and dense (up to $n\left(\mathrm{H}_{2}\right) \simeq 10^{6}-10^{7} \mathrm{~cm}^{-3}$ ) post-shocked gas in $\mathrm{H}_{2}$ Peaks 1 and 2 (e.g., González-Alfonso et al. 2002; Goicoechea et al. 2015b). In our HIFI maps, these extreme conditions can be inferred from the moderately extended emission of the HCN $J=13-12$ line (Fig. B.2), a rotational transition with a critical density close to $10^{10} \mathrm{~cm}^{-3}\left(n_{\mathrm{cr}}=A_{\mathrm{ul}} / \gamma_{\mathrm{ul}}\left(T_{\mathrm{k}}\right)\right.$, where $\gamma_{\mathrm{ul}}$ is the collisional de-excitation rate coefficient in $\mathrm{cm}^{3} \mathrm{~s}^{-1}$ ), around $\mathrm{BN} / \mathrm{KL}$ outflows. Interestingly, the observed $\mathrm{HCN}$ to $\mathrm{HCO}^{+} J=6-5$ line intensity ratio is $\geq 2$ toward $\mathrm{BN} / \mathrm{KL}$, and $<1$ almost elsewhere (see Fig. B.4, right). This may reflect a change in the chemistry between the extended PDR cloud component and the shocked gas in BN/KL outflows. It may also reflect the much stronger mid-IR (MIR) radiation from the $\mathrm{BN} / \mathrm{KL}$ region that favors the radiative pumping of $\mathrm{HCN}$ through its vibrational levels and enhances the high- $J$ rotational emission (e.g, Carroll \& Goldsmith 1981; Ziurys \& Turner 1986). Finally, the maps show that both $\mathrm{HCO}^{+}$and $\mathrm{HCN} J=6-5$ lines display widespread emission outside the main star-forming sites (see Fig. B.1). This suggests that the gas density of the extended cloud layers traced by $\mathrm{HCO}^{+}$and $\mathrm{HCN} J=6-5$ lines is moderately high.
Table 2. Total line integrated luminosities in OMC-1.

\begin{tabular}{lcc}
\hline \hline Line & $L\left(L_{\odot}\right)$ & Surface $L\left(L_{\odot} \mathrm{pc}^{-2}\right)$ \\
\hline$[\mathrm{C}$ II $] 158 \mu \mathrm{m}$ & $1.7 \times 10^{2}$ & $1.4 \times 10^{2}$ \\
$\mathrm{CO} J=10-9$ & $5.1 \times 10^{0}$ & $4.0 \times 10^{0}$ \\
$\mathrm{CO} J=2-1$ & $1.5 \times 10^{-1}$ & $1.2 \times 10^{-1}$ \\
$\mathrm{CH}^{+} J=1-0$ & $1.4 \times 10^{-1}$ & $1.1 \times 10^{-1}$ \\
${ }^{13} \mathrm{CO} J=2-1$ & $2.1 \times 10^{-2}$ & $1.7 \times 10^{-2}$ \\
$\mathrm{C}^{18} \mathrm{O} J=2-1$ & $2.2 \times 10^{-3}$ & $1.7 \times 10^{-3}$ \\
\hline
\end{tabular}

Notes. Within the mapped area of $\sim 7.5^{\prime} \times 11.5^{\prime}(\sim 0.9 \mathrm{pc} \times 1.4 \mathrm{pc})$.

\section{2. $\mathrm{C}^{+}, \mathrm{CO}$ and $\mathrm{CH}^{+}$line luminosities: warm gas cooling}

For collisionally excited transitions and subsequent optically thin emission, the emitted line luminosity ${ }^{3}$ provides a measure of its gas cooling power. The [C II] $158 \mu \mathrm{m}$ line dominates the cooling of FUV-irradiated gas. Indeed, it is the brightest of the observed lines toward OMC-1 (see Table 2), with a surface luminosity of $L_{\mathrm{CII}} / A \simeq 140 L_{\odot} \mathrm{pc}^{-2}$ (Goicoechea et al. 2015a). This is more than three orders of magnitude more luminous than the widely observed low-J CO lines (Bally et al. 1987; Berné et al. 2014). Despite being a trace species, the luminosity emitted by the $\mathrm{CH}^{+} J=1-0$ line in OMC-1 is similar to that of CO $J=2-1$. Remarkably, the CO $J=10-9$ line, with $L_{\mathrm{CO} 10-9} / A \simeq 4 L_{\odot} \mathrm{pc}^{-2}$, is 35 times brighter than the $\mathrm{CO} J=2-1$ line along the mapped region, and it is more than a hundred times brighter toward specific dense PDRs like the Orion Bar. This means that the $\mathrm{CO}$ line cooling of warm molecular gas is dominated by the mid- $J$ lines. In addition, the average ${ }^{12} \mathrm{CO} /{ }^{13} \mathrm{CO} J=2-1$ line luminosity ratio in the map is $\sim 7$; and it is lower than $\sim 15$ in most of the region. This is about an order of magnitude lower than the ${ }^{12} \mathrm{C} /{ }^{13} \mathrm{C}$ isotopic ratio in Orion ( $\sim 67$; Langer \& Penzias 1990) and implies that the ${ }^{12} \mathrm{CO} J=2-1$ line is optically thick at $\sim 1 \mathrm{pc}^{2}$ scales. On the other hand, the high ${ }^{13} \mathrm{CO} / \mathrm{C}^{18} \mathrm{O} J=2-1$ luminosity ratio, $\simeq 9$, indicates that both lines are optically thin at large scales.

Only in shocked and post-shocked gas, such as $\mathrm{H}_{2}$ Peak 1 and Peak 2 regions in $\mathrm{BN} / \mathrm{KL}$ outflows, the hot molecular gas cooling is dominated by emission from FIR high- $J$ CO $(J>15)$ and water vapor lines, and from MIR $\mathrm{H}_{2}$ lines (e.g., González-Alfonso et al. 2002; Goicoechea et al. 2015b).

\subsection{Kinematics of the FUV-irradiated Gas: velocity maps}

Figure 3 shows $\mathrm{CH}^{+}(J=1-0)$ and $\mathrm{CO}(J=10-9)$ velocity channel emission maps, both in color scale, compared to those of [C II] $158 \mu \mathrm{m}$ in white contours. Both panels display the emission from $v_{\mathrm{LSR}}=0$ to $16 \mathrm{~km} \mathrm{~s}^{-1}$ in steps of $2 \mathrm{~km} \mathrm{~s}^{-1}$. The line centroid of the molecular cloud emission lies between $v_{\mathrm{LSR}} \simeq 8.5$ and $10.5 \mathrm{~km} \mathrm{~s}^{-1}$ (e.g., Bally et al. 1987). At the angular resolution of our observations, the $\mathrm{CH}^{+} J=1-0$ and CO $J=10-9$ emission appears at these velocities too. In most channels, there is a very good agreement between the $\mathrm{CH}^{+}(J=1-0)$ and [C II] $158 \mu \mathrm{m}$ emission structures $\left(\mathrm{C}^{+}\right.$main spectral component; see Goicoechea et al. 2015a). The brightest $\mathrm{CH}^{+}$emission peaks arise from the interface between the HII region around the Trapezium and OMC-1. The spatial distribution of these bright

\footnotetext{
3 Line-luminosity $(L)$ computed in power units ( $\mathrm{W}$ or $L_{\odot}$ ). The conversion from velocity-integrated line intensities $W=\int \Delta T_{\mathrm{b}} \mathrm{dv}\left(\mathrm{K} \mathrm{km} \mathrm{s}^{-1}\right)$ to line surface brightness $I$ (in $\mathrm{W} \mathrm{m}^{-2} \mathrm{sr}^{-1}$ ) is $I=2 \mathrm{k} W v^{3} / c^{3}$, with $v$ the line frequency, and $\Delta T_{\mathrm{b}}$ the continuum-subtracted brightness temperature.
} 
peaks suggest a spherical shell structure, at least in projection, where the $\mathrm{H}$ II region is confined by the background dense cloud. The redshifted $\mathrm{CH}^{+}$and $\mathrm{C}^{+}$structures $\left(v_{\mathrm{LSR}}=12\right.$ to $16 \mathrm{~km} \mathrm{~s}^{-1}$; Fig. 3) are likely compressed structures that are being pushed away from the source of FUV radiation, the Trapezium stars, and toward the molecular cloud.

In most areas, the gas kinematics revealed by the CO $J=10-9$ and $\mathrm{CH}^{+} J=1-0$ lines is similar. Toward the more edge-on H II/OMC-1 interfaces, the bright Orion Bar and East PDRs for example, the maps convolved to the same angular resolution show that the CO $J=10-9$ line peaks slightly deeper inside the molecular cloud (Figs. 3 and B.3). Below $v_{\text {LSR }} \simeq 4 \mathrm{~km} \mathrm{~s}^{-1}$ and above $\simeq 16 \mathrm{~km} \mathrm{~s}^{-1}$, the velocity channel maps show CO $J=10-9$ emission only from Orion $\mathrm{S}$ and BN/KL outflows (Fig. 3). In agreement with previous observations (e.g., Tahani et al. 2016, and references therein), there is $\mathrm{CH}^{+} J=1-0$ and $\mathrm{CO} J=10-9$ emission component between $v_{\mathrm{LSR}} \simeq 6$ and $8 \mathrm{~km} \mathrm{~s}^{-1}$ that traces the strongly irradiated surface of the cloud that envelops Orion S. In addition to lie closer to the gravitational center of the ONC (Hacar et al. 2017), Orion S seems to be a condensation detached from OMC-1 and embedded in the $\mathrm{H}$ II region itself (O'Dell et al. 2009). $\mathrm{CH}^{+}(J=1-0)$ is brighter toward Orion $\mathrm{S}$ than toward BN/KL.

Outside these bright emission areas, there is still widespread but fainter $\mathrm{CH}^{+}(J=1-0)$ emission from the extended cloud. We note that some of the spatial distribution differences in the $\mathrm{CH}^{+} J=1-0$ and $\mathrm{CO} J=10-9$ velocity channel maps are due to the systematically broad $\mathrm{CH}^{+} J=1-0$ line-profiles (see next section). Only the emission from hydrogen and helium recombination lines, arising from ionized gas in the $\mathrm{H}$ II region, appears at negative LSR velocities, blueshifted by $\sim 10-20 \mathrm{~km} \mathrm{~s}^{-1}$ with respect to the molecular gas emission. This is the signature of flows of ionized gas that photoevaporate from OMC-1 and toward the observer (e.g., Genzel \& Stutzki 1989; Goicoechea et al. 2015a).

\section{Analysis}

\subsection{Line profiles: broad $\mathrm{CH}^{+}$profiles at large scales}

Based on our knowledge of OMC-1 (Genzel \& Stutzki 1989; Stacey et al. 1993; Bally 2008) and to ease the interpretation of our maps, we select 10 representative positions that we analyze in more detail (Table 3). These positions are marked in the figures with a symbol \#. Figure 4 shows [C II] $158 \mu \mathrm{m}$ (red), CO $J=10-9$ (black), $\mathrm{CH}^{+} J=1-0$ (blue), and $\mathrm{C}^{18} \mathrm{O} J=2-1$ (green) line spectra toward these positions, extracted from maps convolved to a uniform angular resolution of $27^{\prime \prime}(\sim 0.05 \mathrm{pc})$. In Appendix B we show $\mathrm{HCO}^{+} J=6-5$, $\mathrm{HCN} J=6-5$, and $\mathrm{C}^{18} \mathrm{O} J=2-1$ spectra from maps convolved to $43^{\prime \prime}$ (Fig. B.5).

Toward most positions, the observed emission is characterized by a single, roughly Gaussian line profile centered at the LSR velocities of OMC-1. The only exception is the [C II] $158 \mu \mathrm{m}$ line, that shows a second emission component between $v_{\mathrm{LSR}}=-2$ and $+5 \mathrm{~km} \mathrm{~s}^{-1}$ (Goicoechea et al. 2015a). This emission arises from the near side of a 2 pc radius expanding shell of gas driven by the strong winds from star $\theta^{1}$ Ori C1 (Pabst et al. 2019). This foreground component, also known as Orion's Veil, has no, or little, molecular line emission. Finally, all species that are abundant in shocked gas $\left(\mathrm{CO}, \mathrm{H}_{2} \mathrm{O}, \mathrm{HCO}^{+}, \mathrm{HCN}\right.$, etc.) display high-velocity wings toward Orion $\mathrm{S}$ and $\mathrm{BN} / \mathrm{KL}$ (see spectra of position \#9 in Figs. 4 and B.5).

$\mathrm{C}^{+}$and $\mathrm{CH}^{+}$line profiles show a slightly different behavior among them. While the [C II] $158 \mu \mathrm{m}$ line is narrow toward
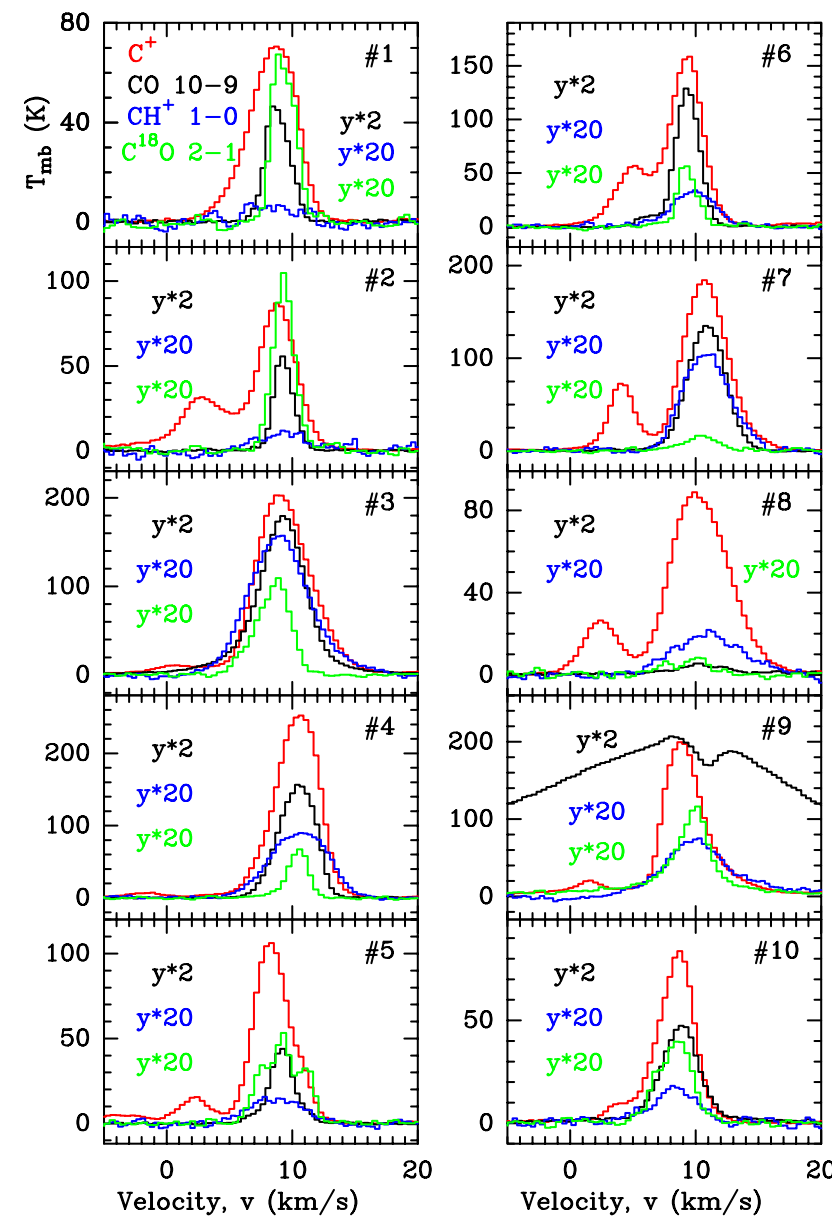

Fig. 4. Spectra at representative positions (see Table 3). Lines were extracted from maps convolved to the same angular resolution of $27^{\prime \prime}$.

Table 3. Representative positions discussed in the text.

\begin{tabular}{lcl}
\hline \hline Position & Offset & Comments \\
\hline$\# 1$ & $\left(76^{\prime \prime}, 305^{\prime \prime}\right)$ & Northern ridge \\
$\# 2$ & $\left(-25^{\prime \prime}, 178^{\prime \prime}\right)$ & North-west ridge edge \\
$\# 3$ & $\left(-38^{\prime \prime},-50^{\prime \prime}\right)$ & CH $^{+}$peak near Trapezium \\
$\# 4$ & $\left(51^{\prime \prime},-165^{\prime \prime}\right)$ & Orion Bar PDR \\
$\# 5$ & $\left(-25^{\prime \prime},-291^{\prime \prime}\right)$ & Southern edge \\
$\# 6$ & $\left(51^{\prime \prime}, 166^{\prime \prime}\right)$ & Northern lane \\
$\# 7$ & $\left(114^{\prime \prime}, 13^{\prime \prime}\right)$ & East PDR \\
$\# 8$ & $\left(76^{\prime \prime},-63^{\prime \prime}\right)$ & Toward dense H II region \\
\#9 & $\left(-25^{\prime \prime}, 13^{\prime \prime}\right)$ & BN-KL outflows \\
$\# 10$ & $\left(-114^{\prime \prime}, 1^{\prime \prime}\right)$ & Western edge \\
\hline
\end{tabular}

$\mathrm{BN} / \mathrm{KL}$ outflows, the $\mathrm{CH}^{+} \quad J=1-0$ profile displays a weak red-wing emission. This demonstrates that the outflows around $\mathrm{BN} / \mathrm{KL}$ are illuminated by a moderate FUV radiation field (Chen et al. 2014; Goicoechea et al. 2015b; Melnick \& Kaufman 2015). It has been suggested that the available $\mathrm{C}^{+}$in irradiated dense shocked gas must be quickly converted into $\mathrm{CH}^{+}$ (Morris et al. 2016). Nevertheless, the $\mathrm{CH}^{+} J=1-0$ wing is faint compared to $\mathrm{CO}$ or $\mathrm{H}_{2} \mathrm{O}$ lines, and is only restricted to low velocities.

Table A.1 tabulates the velocity centroid, obtained from Gaussian fits, toward the ten representative positions. All lines show, within fit errors and spectral resolution, similar velocity 


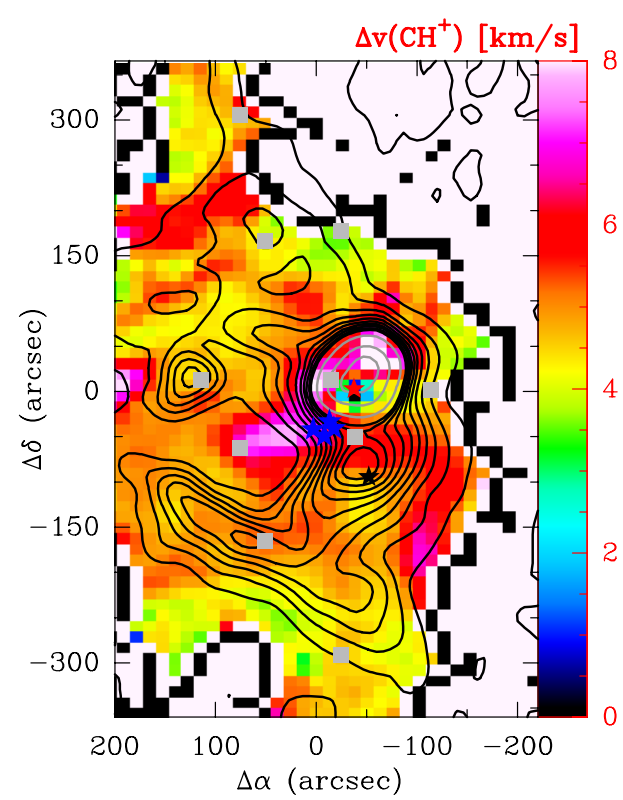

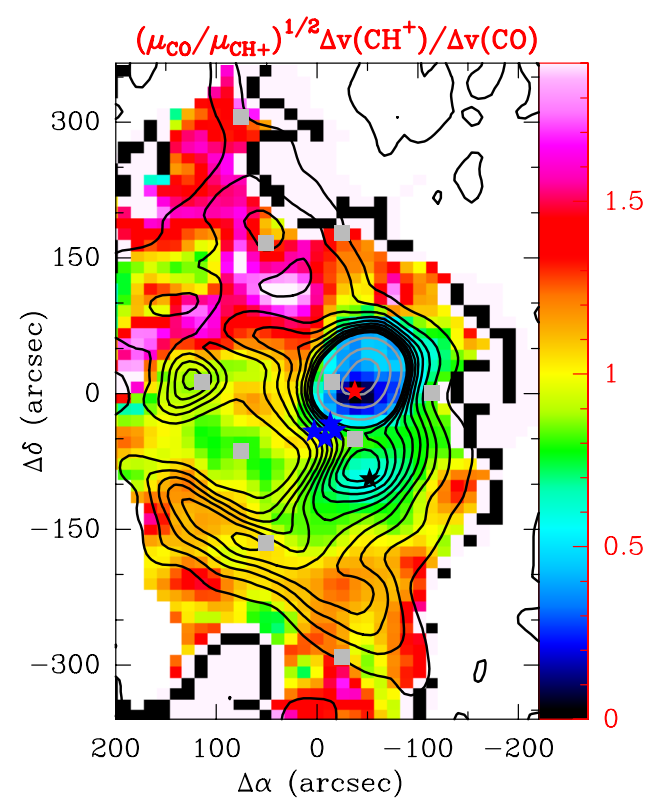

Fig. 5. Left panel: map of the $\mathrm{CH}^{+}$ $J=1-0$ linewidths in $\mathrm{km} \mathrm{s}^{-1}$ shown in color scale. Right panel: map of the $\mathrm{CH}^{+}(J=1-0)$ to $\mathrm{CO}(J=10-9)$ linewidth ratio, in color scale, corrected by the different molecular masses. In both panels, black and gray contours show the CO $J=10-9$ line integrated intensity. All maps were convolved to the same $27^{\prime \prime}$ angular resolution. centroids. Table A.2 tabulates the linewidth for the same set of lines and positions; there is more scatter in this case. In principle, the gas velocity dispersion is determined by the gas temperature, through the thermal broadening caused by non-reactive collisions, and by gas macroscopic motions: turbulence, outflows, etc. The narrowest measured widths $\left(\Delta v<3 \mathrm{~km} \mathrm{~s}^{-1}\right)$ are those of $\mathrm{C}^{18} \mathrm{O}$ lines, predominantly arising from FUV-shielded and colder gas. At large spatial scales, the CO $J=10-9$ line also displays narrow linewidths $\left(\Delta v \simeq 3 \mathrm{~km} \mathrm{~s}^{-1}\right)$ except toward $\mathrm{BN} / \mathrm{KL}$ and Orion $\mathrm{S}$ outflows. This confirms that the bulk of the mid- $J \mathrm{CO}$ emission arises from relatively quiescent gas and not from fast shocks (see also Wilson et al. 2001; Peng et al. 2012).

Assuming optically thin emission and negligible nonthermal line broadening, the observed CO $J=10-9$ linewidth sets an upper limit value to the emitting-gas temperature of $T_{\mathrm{k}} \lesssim 450 \mathrm{~K}$. Taking a more realistic non-thermal velocity dispersion of $\sigma_{\text {nth }}=1 \mathrm{~km} \mathrm{~s}^{-1}\left(\Delta v_{\text {nth,FHWM }}=2.355 \cdot \sigma_{\text {nth }}\right.$, where FHWM refers to the full width at half maximum) for the mid- $J$ CO-emitting PDR gas (see next section), one obtains $T_{\mathrm{k}} \lesssim 150 \mathrm{~K}$. These values are consistent with the lower limit to the gas temperature provided by the $\mathrm{CO} J=2-1$ line intensity peak $\left(T_{\text {peak }}\right.$ in $\mathrm{K}$; see map in Fig. C.1). Because the CO $J=2-1$ emission is optically thick in most of the field, one obtains $T_{\text {peak }} \simeq T_{\text {rot }}$ $(\mathrm{CO} 2-1) \leq T_{\mathrm{k}}$.

The observed [C II] $158 \mu \mathrm{m}$ line-peak temperatures, however, suggest that the $\mathrm{C}^{+}$-emitting layers, the atomic PDR, are hotter ( $T_{\mathrm{k}} \geq 300 \mathrm{~K}$; Goicoechea et al. 2015a) than the CO-emitting layers located slightly deeper into the PDR (see model predictions in Fig. 10). This difference is the signature of sharp temperature gradients: from the H II region, the atomic PDR and molecular PDR, to the shielded cloud interior.

$\mathrm{CH}^{+}(J=1-0)$ shows the broadest linewidths over the observed field, broader than [C II] $158 \mu \mathrm{m}$ and CO $J=10-9$ lines. Figure 5 (left) shows a map of the $\mathrm{CH}^{+} J=1-0$ linewidths in the region. Unlike other species, the broad $\mathrm{CH}^{+} J=1-0$ line-profiles are quite uniform along the mapped area $\left(\Delta v \simeq 5-6 \mathrm{~km} \mathrm{~s}^{-1}\right)$. Figure 5 (right) shows a map of the $\mathrm{CH}^{+}(J=1-0)$ to $\mathrm{CO}(J=10-9)$ linewidth ratio corrected by their different molecular masses, $\left(\mu_{\mathrm{CO}} / \mu_{\mathrm{CH}^{+}}\right)^{1 / 2}=1.47$. Only toward $\mathrm{BN} / \mathrm{KL}$ and Orion $\mathrm{S}$ outflows, the ratio is much lower than one. In the extended cloud component, the linewidth ratio is always greater than one. These maps thus confirm the broader $\mathrm{CH}^{+}$line profiles, not only toward local bright and dense H II/PDR interfaces (Nagy et al. 2013; Morris et al. 2016; Parikka et al. 2017) but at large-spatial scales, where the $\mathrm{CH}^{+}$ $(J=1-0)$ emission is certainly optically thin and not affected by opacity broadening. Taking $\sigma_{\text {nth }}=1 \mathrm{~km} \mathrm{~s}^{-1}$, the observed broad $\mathrm{CH}^{+} \quad J=1-0$ linewidths would imply unrealistically high gas temperatures, from $T_{\mathrm{k}} \simeq 5500-8600 \mathrm{~K}$. As previously discussed in the literature (Black 1998; Nagy et al. 2013; Godard \& Cernicharo 2013; Goicoechea et al. 2017), this broadening is likely related to the high reactivity of the ion, and to the exothermic route $(\Delta E / k \geq 5360 \mathrm{~K})$ that forms $\mathrm{CH}^{+}$from reaction $\mathrm{C}^{+}+\mathrm{H}_{2}(v \geq 1)$. The $\mathrm{CH}^{+}$lifetime in dense PDRs is so short that the newly formed molecular ion does not have time to thermalize its translational motions through elastic collisions and to a Maxwellian velocity distribution at $T_{\mathrm{k}}$. In this interpretation, part of the formation exothermicity goes into excitation and translational motion. Hence, the broad $\mathrm{CH}^{+}$linewidths would be related to the excess of energy upon formation (Nagy et al. 2013). We see that in high-mass star-forming regions like OMC-1, sources of strong FUV radiation, this mechanism operates at large-spatial scales.

\subsection{Physical conditions of the extended warm gas}

In this section we estimate the average physical conditions of the extended warm molecular gas traced by the observed submm lines. For high-dipole molecules such as $\mathrm{HCO}^{+}$, having high critical density transitions, the intensity ratio of two rotational lines is a good tracer of gas density variations. On the other hand, for low-dipole moment molecules such as $\mathrm{CO}$, and for gas densities comparable or higher than the critical density, line intensity ratios trace gas temperature variations.

Figure 6 shows maps of the $\mathrm{HCO}^{+} 6-5 / 3-2$ and $\mathrm{CO} 10-9 /$ 2-1 line intensity ratios, obtained from integrated intensities in units of $\mathrm{W} \mathrm{m}^{-2} \mathrm{sr}^{-1}$. To invert the observed ratios into a range of beam-averaged gas temperatures, $T_{\mathrm{k}}$, and densities, $n_{\mathrm{H}}=n(\mathrm{H})+$ $2 n\left(\mathrm{H}_{2}\right)$, we compare the observed line intensities with predictions of a grid of non-local and non-LTE excitation models (Monte Carlo radiative transfer code of Goicoechea et al. 2006). 

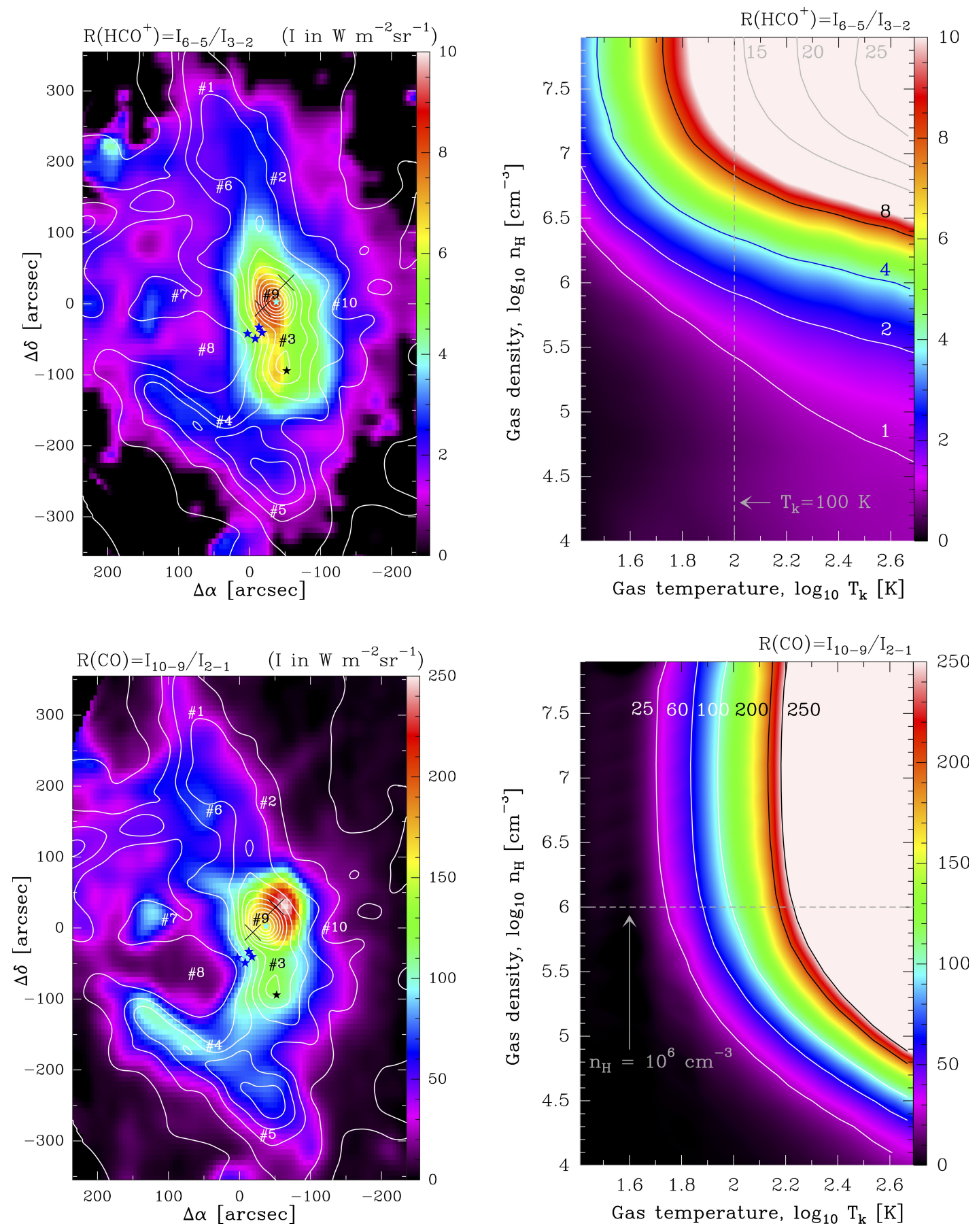

Fig. 6. Top left panel: map of the $\mathrm{HCO}^{+} 6-5 / 3-2$ intensity ratio, at $43^{\prime \prime}$ resolution, sensitive to gas density variations. Bottom left panel: map of the CO 10-9/2-1 intensity ratio, at $27^{\prime \prime}$ resolution, sensitive to gas temperature variations. In both maps the white contours represent the ${ }^{13} \mathrm{CO} J=2-1$ optically thin emission. Right panel: grid of non-local and non-LTE excitation models and predicted line intensity ratios.

The model accounts for line trapping, collisional excitation, and radiative excitation by absorption of the $2.7 \mathrm{~K}$ cosmic background and by the dust continuum emission. In FUV-illuminated gas, the high electron abundance, up to $x_{e}=n_{e} / n_{\mathrm{H}} \lesssim x\left(\mathrm{C}^{+}\right) \simeq$ $10^{-4}$ for standard cosmic ray ionization rates, can play an important role in the collisional excitation of high-dipole moment molecules as the collisional rates compete with those of $\mathrm{H}_{2}$ and H (e.g., van der Tak et al. 2012; Goldsmith \& Kauffmann 2017). Here we assume an ionization fraction of $x_{e}=10^{-4}$ (roughly the $\mathrm{C}^{+}$abundance in PDR gas), which means that our derived $n_{\mathrm{H}}$ values are lower limits if $x_{e}$ is not that high. We used $\mathrm{HCO}^{+}-$ $\mathrm{H}_{2}$ and $\mathrm{HCO}^{+}-e^{-}$inelastic collisional rates from Flower (1999), Faure \& Tennyson (2001), and Fuente et al. (2008). For CO, we just used the rates for $\mathrm{CO}-\mathrm{H}_{2}$ collisions from Yang et al. (2010).
Regarding radiative excitation, we modeled the frequencydependent dust continuum emission as a modified black body with an effective dust grain temperature of $T_{\mathrm{d}}=55 \mathrm{~K}$, spectral emissivity index of 2 , and a dust opacity $\tau_{\mathrm{d}}=0.05$ at a reference wavelength of $160 \mu \mathrm{m}$. These FIR and submm illumination conditions are typical of the Orion Bar PDR (Arab et al. 2012) but we note that submm pumping plays a negligible role in the excitation of the extended $\mathrm{CO}$ and $\mathrm{HCO}^{+}$emission. This is, however, not the case of $\mathrm{H}_{2} \mathrm{O}$ or $\mathrm{OH}$ excitation toward $\mathrm{BN} / \mathrm{KL}$, where the dust thermal continuum is very strong (e.g., González-Alfonso et al. 2002; Melnick et al. 2010; Goicoechea et al. 2015b). Our model includes thermal, turbulent, and line opacity broadening. The non-thermal velocity dispersion that reproduces the observed linewidths is typically $\sigma_{\text {nth }} \simeq 1 \mathrm{~km} \mathrm{~s}^{-1}$. We adopted column densities of $N(\mathrm{CO})=5 \times 10^{17} \mathrm{~cm}^{-2}$ and 
$N\left(\mathrm{HCO}^{+}\right)=5 \times 10^{13} \mathrm{~cm}^{-2}$ extracted from our mm line survey toward the edge of the Orion Bar (e.g., Cuadrado et al. 2015; Goicoechea et al. 2017).

Figure 6 (right panels) shows synthetic line ratios in the plane $\log \left(T_{\mathrm{k}}\right)-\log \left(n_{\mathrm{H}}\right)$, with same color scale as in the maps, for a grid of models with $n_{\mathrm{H}}$ ranging from $10^{4}$ to $10^{8} \mathrm{~cm}^{-3}$, and $T_{\mathrm{k}}$ from 25 to $500 \mathrm{~K}$. The lowest measured $\mathrm{HCO}^{+} 6-5 / 3-2$ intensity ratio in the map is $\sim 1$ (magenta regions, e.g., positions \#1 and \#5). This ratio imposes a minimum gas density of a few $10^{5} \mathrm{~cm}^{-3}$ for $T_{\mathrm{k}} \lesssim 150 \mathrm{~K}$. Bright edge-on H II/OMC-1 interfaces such as the Orion Bar (position \#4) or the East PDR (position \#7) require higher gas temperatures and densities $\left(T_{\mathrm{k}} \simeq\right.$ $100 \mathrm{~K}$ and $\left.n_{\mathrm{H}} \simeq 10^{6} \mathrm{~cm}^{-3}\right)$. Closer to the Trapezium cluster and around Orion $\mathrm{S}$ (position \#3), densities increase (reaching $\sim 10^{7} \mathrm{~cm}^{-3}$ ). The shocked gas associated with Orion BN/KL (position \#9) shows the highest excitation conditions in both $T_{\mathrm{k}}$ and $n_{\mathrm{H}}$, but we stress again that most of the line integrated emission in the map arises from the irradiated cloud surface. This is an extended but thin gas layer (see PDR models in Sect. 5.1) characterized by high thermal pressures, in the range $P_{\text {th }}=T_{k} \cdot n_{\mathrm{H}} \approx 10^{7}-10^{9} \mathrm{~cm}^{-3} \mathrm{~K}$ according to our models. The presence of a layer of high-density gas close to the interface between the $\mathrm{H}$ II region and the molecular cloud was anticipated by Rodríguez-Franco et al. $(1998,2001)$ from observations of the $\mathrm{CN}$ radical, which predominantly arises, but not only (e.g., Pety et al. 2017), from PDR gas. They also estimated gas densities ranging from $\sim 10^{5} \mathrm{~cm}^{-3}$ toward the extended cloud, to several $\sim 10^{6} \mathrm{~cm}^{-3}$ toward the Trapezium region. They could not, however, constrain the gas temperatures.

Using our $\mathrm{CO}$ maps, we estimate the mass contained in the high-pressure molecular $\mathrm{PDR}$ layer, $M_{\mathrm{mPDR}}\left(\mathrm{H}_{2}\right)$, along OMC-1. Assuming $T_{\text {rot }}(10-9) \simeq T_{\text {rot }}(2-1)$ (see map in Fig. C.1) and that the $\mathrm{CO} J=10-9$ emission is optically thin and only arises from the cloud surface, we convert the CO $J=10-9$ integrated intensity map into a $\mathrm{CO}$ column density map, $N(\mathrm{CO})$, and then into a total column density map as $N_{\mathrm{H}}=x(\mathrm{CO}) \cdot N(\mathrm{CO}) \simeq 10^{-4} \cdot N(\mathrm{CO})$ (see Appendix C for details). With these assumptions, we obtain $M_{\mathrm{mPDR}}\left(\mathrm{H}_{2}\right) \simeq 150 M_{\odot}$. Allowing a opacity correction factor toward lines of sight with $\tau_{10-9} \simeq 1$, we then derive $M_{\mathrm{mPDR}}\left(\mathrm{H}_{2}\right) \simeq 300 M_{\odot}$. This warm molecular gas mass is similar to the $\sim 200 M_{\odot}$ mass that arises from the [C II] $158 \mu \mathrm{m}$ emitting gas (the atomic PDR; Goicoechea et al. 2015a). It represents between $\sim 5$ and $10 \%$ of the total gas mass in OMC-1 (Goicoechea et al. 2015a). Finally, using $A_{\mathrm{V}} / N_{H}=3.5 \times 10^{-22} \mathrm{mag} \mathrm{cm}{ }^{2}$, appropriate to Orion, we derive that the average extinction thickness traced by the $\mathrm{CO}$ $J=10-9$ line (roughly the high-pressure cloud-surface layer) is $\left\langle A_{\mathrm{V}}\right\rangle \simeq 3-6 \mathrm{mag}$.

\section{3. $\mathrm{CH}^{+} \mathrm{J}=1-0$ intensity correlation plots}

Figure 7 shows spatial correlation plots between the $\mathrm{CH}^{+}$ $J=1-0$ line intensity and other lines or quantities mapped along OMC-1: (a) the strength of the FUV radiation flux, $G_{0}$, estimated by Goicoechea et al. (2015a) from FIR luminosities in the region, (b) $[\mathrm{C}$ II $] 158 \mu \mathrm{m}$, (c) $\mathrm{CO} J=10-9$, and (d) $\mathrm{C}^{18} \mathrm{O} J=2-1$ line intensities (all in $\mathrm{W} \mathrm{m}^{-2} \mathrm{sr}^{-1}$ ). The correlation plots are shown in a linear scale (cyan points). The results from a linear fit are shown in blue. The small inset in each panel shows the same data in $\log -\log$ scale (gray points). These plots use all the line intensity measurements contained in the maps and thus provide robust clues to the origin of the $\mathrm{CH}^{+}$emission at large scales.

Owing to the proximity of the Trapezium stars, $G_{0}$ is very high along OMC-1 $\left(G_{0} \simeq 10^{3}-10^{5}\right)$. Under these irradiation conditions, the $\mathrm{CH}^{+} J=1-0$ line intensity appears tightly correlated with $G_{0}$ (Fig. 7a). The $\mathrm{CH}^{+}(J=1-0)$ emission also scales with [C II] $158 \mu \mathrm{m}$ line intensity (Fig. 7b). This shows again that $\mathrm{CH}^{+}$arises very close to the edge of the molecular cloud. Indeed, the $\mathrm{CH}^{+}$emission is much less correlated with the $\mathrm{C}^{18} \mathrm{O} J=2-1$ emission that, at first order, traces the column density in the colder cloud interior (Fig. 7d). Toward positions such as the Orion Bar PDR, where the [C II] $158 \mu \mathrm{m}$ line is very bright: $I([\mathrm{C} \mathrm{II}]) \gtrsim 8 \times 10^{-6} \mathrm{~W} \mathrm{~m}^{-2} \mathrm{sr}^{-1}$, the $\mathrm{C}^{+}$versus $\mathrm{CH}^{+}$correlation starts to decline. Observations of the ${ }^{13} \mathrm{C}^{+}$finestructure lines show that the [C II] $158 \mu \mathrm{m}$ line actually becomes optically thick toward very bright PDRs (Ossenkopf et al. 2013; Goicoechea et al. 2015a). This may partially explain that the $\mathrm{C}^{+}$ versus $\mathrm{CH}^{+}$relation becomes less linear when the $[\mathrm{C}$ II $] 158 \mu \mathrm{m}$ emission is very bright and opaque. In addition, for moderate densities $\left(n_{\mathrm{H}} \gtrsim 10^{4} \mathrm{~cm}^{-3}\right)$ and FUV radiation fields $\left(G_{0} \lesssim 10^{5}\right)$, the intensity of the [C II] $158 \mu \mathrm{m}$ line is governed by the $G_{0} / n_{\mathrm{H}}$ ratio (e.g., Kaufman et al. 1999). This implies that, in this range of paramenters, the $\mathrm{CH}^{+}(J=1-0) /[\mathrm{C} \mathrm{II}] 158 \mu \mathrm{m}$ intensity ratio would scale with $n_{\mathrm{H}}$. Figure B.4 (left) shows a map of this intensity ratio. Indeed, the brightest regions in the plot are associated with the highest density PDR layers in the region, $n_{\mathrm{H}} \gtrsim 10^{6} \mathrm{~cm}^{-3}$ (also revealed by the $\mathrm{HCO}^{+} 6-5 / 3-2$ map in Fig. 6). Hence, the decline of the [C II] $158 \mu \mathrm{m}$ versus $\mathrm{CH}^{+}(J=1-0)$ emission trend is likely driven by the increase of gas density toward these cloud edges.

Finally, if one excludes the bright CO $J=10-9$ line emission from shocked gas in outflows, that is, positions with $I_{\mathrm{CO} 10-9} \gtrsim 10^{-6} \mathrm{~W} \mathrm{~m}^{-2} \mathrm{sr}^{-1}$ in our maps, there is also a good correlation between $\mathrm{CH}^{+} J=1-0$ and $\mathrm{CO} J=10-9$ line intensities (Fig. 7c). This is another proof of the FUV-irradiated cloud edge origin of the large scale CO $J=10-9$ emission.

\section{4. $\mathrm{CH}^{+}$and the extended FUV-pumped $\mathrm{H}_{2}$ emission}

Right panel in Fig. 8 shows the tight spatial correlation between the distribution of the $\mathrm{CH}^{+}(J=1-0)$ emission (black contours) and that of $G_{0}$ (in color scale). The strongest FUV fluxes $\left(G_{0} \simeq 10^{5}\right)$ appear near Orion $\mathrm{S}$, close to the Trapezium cluster. The Orion Bar and East PDRs are also strongly illuminated $\left(G_{0}\right.$ of a few $\left.10^{4}\right)$. At the edges of the mapped area, the FUV radiation field is still high $\left(G_{0} \approx 10^{3}\right)$. Therefore, an intense stellar FUV flux reaches parsec scales in OMC-1 (Stacey et al. 1993; Goicoechea et al. 2015a). These FUV photons radiatively pump $\mathrm{H}_{2}$ molecules to vibrationally excited states (e.g., Hollenbach \& Tielens 1997, and references therein) at the edge of the irradiated cloud, leading to bright near-IR (NIR) $\mathrm{H}_{2}(v \geq 1)$ emission that is detected at large-spatial scales (Luhman et al. 1994). In particular, $\mathrm{H}_{2}$ lines from vibrational levels up to $v=10$ (or $E_{\mathrm{u}} / k \approx 50000 \mathrm{~K}$ ) have been detected toward the Orion Bar (e.g., Kaplan et al. 2017).

Among the brightest NIR $\mathrm{H}_{2}(v \geq 1)$ lines is the $\mathrm{H}_{2} v=1-0$ $S$ (1) ro-vibrational line. Figure 8 (left) shows a photometric image of OMC-1 taken with the ESO?s Visible and Infrared Survey for Astronomy (VISTA) in the $K_{S}$ band (Meingast et al. 2016). The $K_{S}$ filter is centered at $\lambda=2.15 \mu \mathrm{m}$ and has a width of $\Delta \lambda=0.3 \mu \mathrm{m}$; and thus covers the $\mathrm{H}_{2} v=1-0 \mathrm{~S}(1)(2.12 \mu \mathrm{m})$ and $v=2-1 S(1)(2.24 \mu \mathrm{m})$ lines. Hence, in addition to the hundred of NIR (proto)stellar point-sources in the field, this image is sensitive to extended emission of interstellar $\mathrm{H}_{2}(v \geq 1)$. Hot shocked gas from protostellar outflows also produce bright, collisionally excited, and nearly thermal $\mathrm{H}_{2}(v \geq 1)$ emission locally (e.g., Rosenthal et al. 2000). The most obvious examples in Fig. 8 (left) are $\mathrm{H}_{2}$ Peaks 1 and 2 regions in $\mathrm{BN} / \mathrm{KL}$, around position \#9 

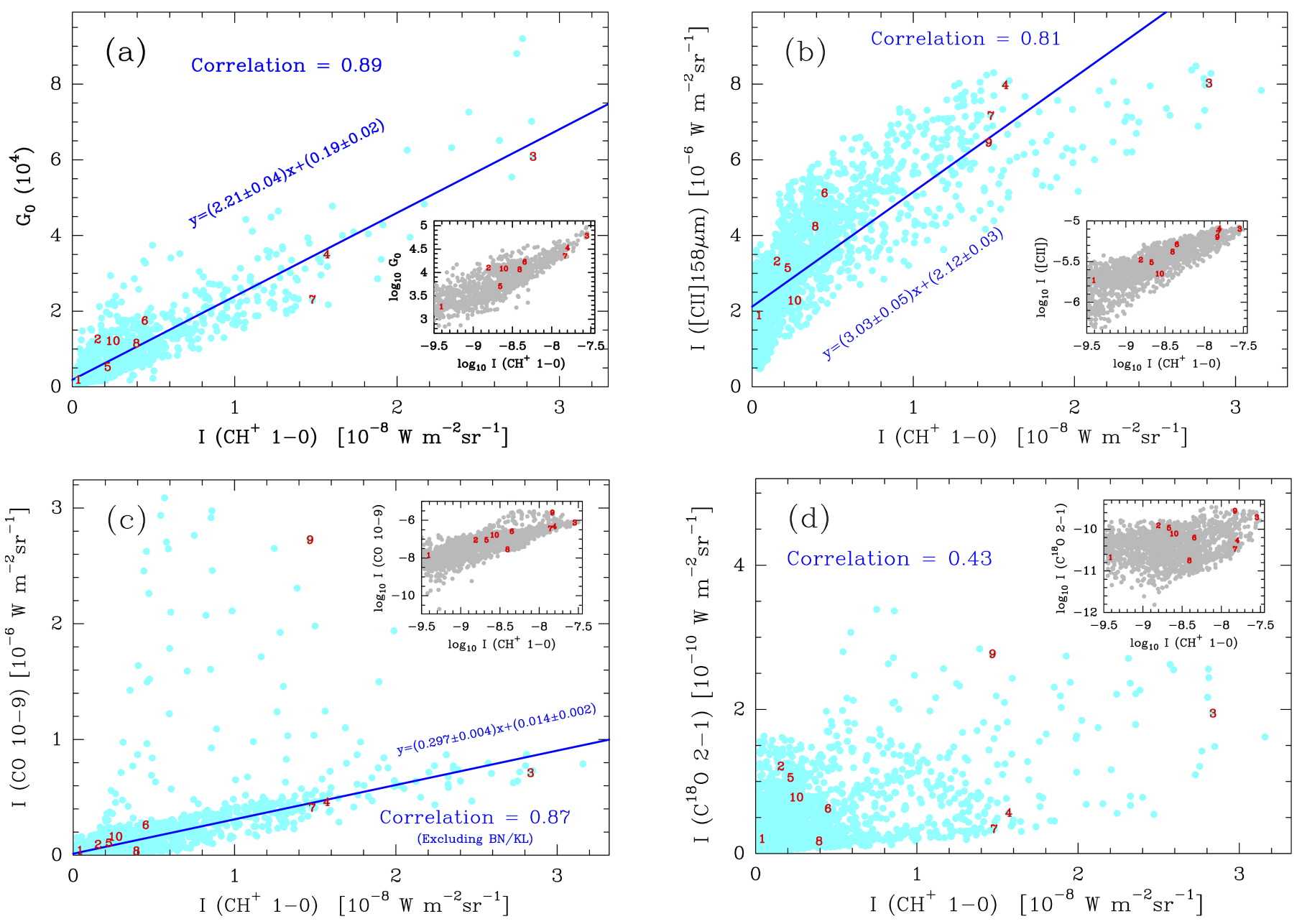

Fig. 7. $\mathrm{CH}^{+} J=1-0$ line intensity correlation plots extracted from maps convolved to a uniform resolution of $27^{\prime \prime}$. Data points are shown in linear scale (cyan points) and in logarithmic scale (gray points). Results and dispersions from a linear fit, $y=(m \pm \Delta m) x+(b \pm \Delta b)$, are shown in blue. The location of selected positions \# in the map (Table 3 ) are indicated with red numbers. Panel $a$ : FUV-radiation field flux $\left(\mathrm{G}_{0}\right)$ estimated from FIR luminosities (Goicoechea et al. 2015a). Panel b: [C II] $158 \mu \mathrm{m}$. Panel c: CO $J=10-9$. Panel $d$ : $\mathrm{C}^{18} \mathrm{O} J=2-1$ line intensities.

in the maps (P1 and P2 in Fig. 1; see also Bally et al. 2011, and references therein). At large scales, however, the $K_{S}$ band image is dominated by extended emission from FUV-pumped $\mathrm{H}_{2}(v \geq 1)$ (e.g., Luhman et al. 1994).

PDR models predict that at moderate gas densities, $n_{\mathrm{H}}>10^{5} \mathrm{~cm}^{-3}$, the FUV-pumping contribution to the $\mathrm{H}_{2} v=1-0$ $S$ (1) line intensity scales with $G_{0}$ (e.g., Burton et al. 1990). Only if the gas temperature is $T_{\mathrm{k}}>1000 \mathrm{~K}$, collisions will also contribute to populate the vibrational level $v=1$, so we expect that thermal excitation does not dominate the large-scale IR $\mathrm{H}_{2}(v \geq 1)$ emission. Hence, in a first approximation, the extended interstellar emission shown in the $K_{S}$ image (Fig. 8, left panel) should also reflect variations of $G_{0}$ along the FUV-irradiated surface of OMC-1. The two images in Fig. 8 display the $\mathrm{CH}^{+}$ $J=1-0$ integrated intensity map in black contours. Although the NIR and submm observations have very different angular resolutions, one clearly infers the presence of $\mathrm{CH}^{+}$everywhere $\mathrm{H}_{2}(v \geq 1)$ emits. Indeed, the brightest $\mathrm{CH}^{+}(J=1-0)$ emission peaks, associated to regions of high $G_{0}$ values, are also bright in NIR $\mathrm{H}_{2}(v \geq 1)$ emission (Fig. 8). The observed spatial correlations reflect the tight connection between both the $\mathrm{CH}^{+}$ rotational and $\mathrm{H}_{2}(v \geq 1)$ ro-vibrational emission with the flux of stellar FUV photons.

\subsection{Rotationally warm $\mathrm{CH}^{+}$emission toward the Trapezium}

In terms of FUV irradiation, the most extreme conditions are those in the regions between the Trapezium and Orion $S$, with $G_{0} \simeq 10^{5}$ (Fig. 8, right). These are even harsher conditions than in the prototypical PDR the Orion Bar (position \#4). Indeed, with Herschel/PACS we detect $\mathrm{CH}^{+}$rotational lines up to $J=5-4$ (or $E_{\mathrm{u}} / k \simeq 600 \mathrm{~K}$ ) toward position \#3. The intensities of these rotationally excited $\mathrm{CH}^{+}$lines (a few $10^{-7} \mathrm{~W} \mathrm{~m}^{-2} \mathrm{sr}^{-1}$ ) are a factor of 4-8 brighter than toward the Orion Bar (Nagy et al. 2013; Joblin et al. 2018). $\mathrm{CH}^{+}$rotational lines have very high critical densities, several $10^{9}-10^{10} \mathrm{~cm}^{-3}$. Hence, at the gas densities we infer for the edge of OMC-1 (Sect. 4.2), one would expect very subthermal excitation; $n_{\mathrm{H}} \ll n_{\text {cr }}$ implies weak collisional excitation, in other words, very low rotational temperatures, $T_{\text {rot }} \ll T_{\mathrm{k}}$, lower than those inferred for reactive ions such as $\mathrm{CO}^{+}$or $\mathrm{HOC}^{+}$ ( $T_{\text {rot }} \simeq 10-30 \mathrm{~K}$ in the Orion Bar; see Fuente et al. 2003; Nagy et al. 2013; Goicoechea et al. 2017). Figure 9 shows the observed $\mathrm{CH}^{+}$line intensities toward position \#3 in the form of a rotational population diagram. Due to the observed curvature of the diagram, the measured line intensities are incompatible with a single rotational temperature. Just for reference, we note that a simple two-temperature fit provides $T_{\text {rot }}\left(\mathrm{CH}^{+}\right) \simeq 67$ and $104 \mathrm{~K}$, significantly higher than $T_{\text {rot }}$ of other reactive ions. Hence, $\mathrm{CH}^{+}$ 

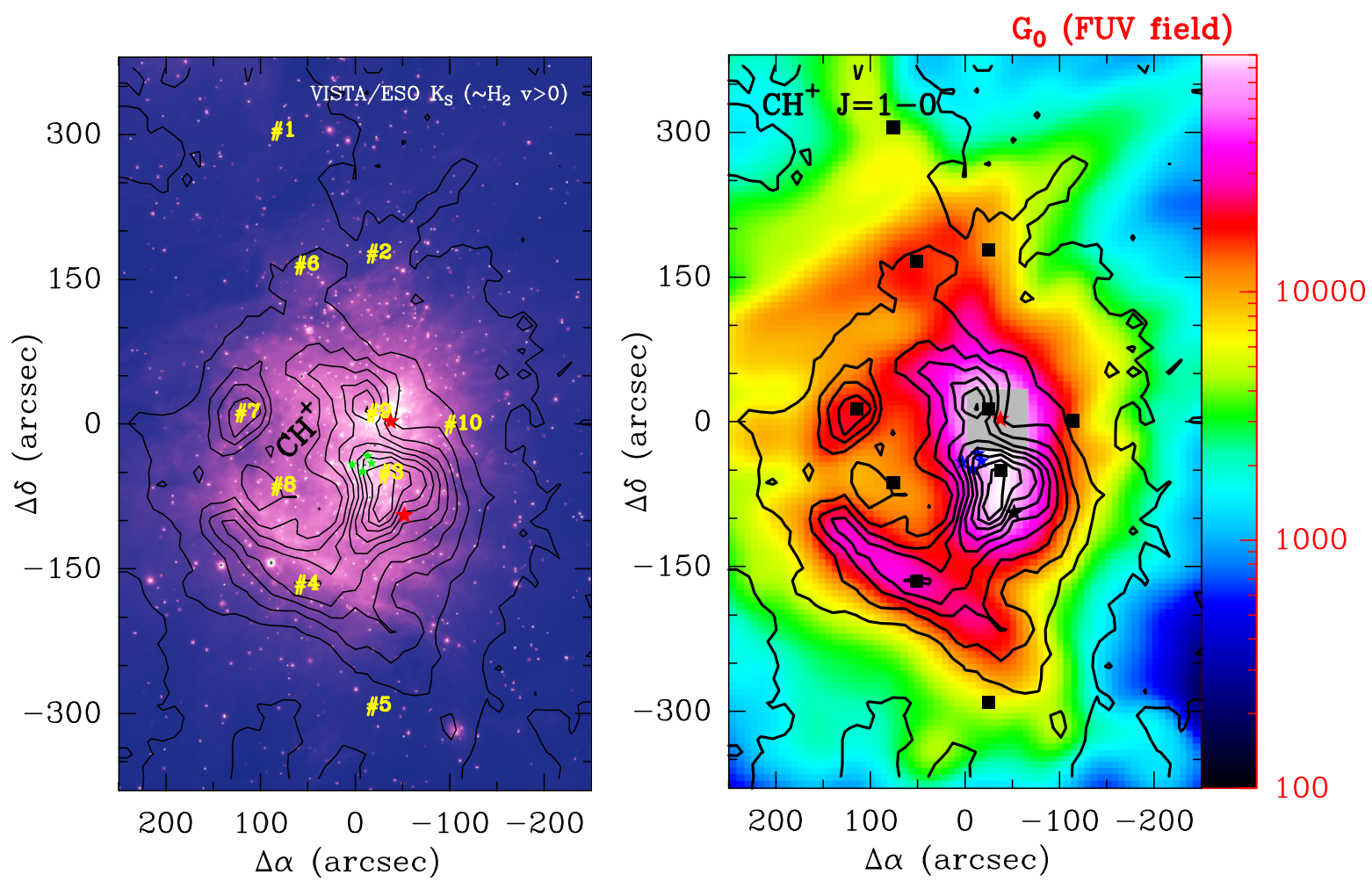

Fig. 8. Left panel: NIR VISTA image of M42 in the $K_{S}$ band dominated by interstellar emission from vibrationally excited $\mathrm{H}_{2}$. The intensity scale is chosen to enhance the very bright NIR emission from gas illuminated by the strong FUV field from the Trapezium stars; and locally by thermally excited shocked gas in outflows (Fig. B.6 shows a larger image that enhances the much more extended NIR emission). Black contours show the $\mathrm{CH}^{+} J=1-0$ intensities from 1 to $46 \mathrm{~K} \mathrm{~km} \mathrm{~s}^{-1}$ in steps of $5 \mathrm{~K} \mathrm{~km} \mathrm{~s}^{-1}$. Right panel: FUV radiation field, $G_{0}$, estimated from FIR luminosities (Goicoechea et al. 2015a). The gray area around BN/KL indicates positions where the Herschel photometric data is saturated.

is rotationally warm. This is consistent with the very short-life of $\mathrm{CH}^{+}$in dense gas and with the exothermic formation pathway from the reaction of $\mathrm{C}^{+}$with $\mathrm{H}_{2}(v \geq 1)$. In particular, $\mathrm{CH}^{+}$can be excited by radiation many times during its short lifetime, and during its mean-free-time for inelastic collisions, so that it remains rotationally warm while it emits (Black 1998; Goicoechea et al. 2017). This formation pumping enhances the populations of $\mathrm{CH}^{+}$ excited levels (especially for $J>1$; Godard \& Cernicharo 2013; Faure et al. 2017).

\section{Discussion}

ALMA observations of the Orion Bar have spatially resolved the compressed PDR layers at the irradiated edge of the cloud, very close to the $\mathrm{H}$ to $\mathrm{H}_{2}$ transition zone. This layer is characterized by high thermal pressures $\left(P_{\mathrm{th}}=n_{\mathrm{H}} \cdot T_{\mathrm{k}} \approx 10^{8} \mathrm{~cm}^{-3} \mathrm{~K}\right.$; Goicoechea et al. 2016; Joblin et al. 2018), it is the source of $\mathrm{H}_{2}(v \geq 1)$ emission, and the only layers where reactive ions such as $\mathrm{CH}^{+}$and $\mathrm{SH}^{+}$can efficiently form, and indeed are observed (Nagy et al. 2013; Goicoechea et al. 2017; Parikka et al. 2017; Joblin et al. 2018). Observations of different PDRs in the Milky Way further suggest a correlation between $G_{0}$ and the thermal pressure in these compressed layers. In particular, many PDRs seem to lie in the range $P_{\mathrm{th}} / G_{0} \approx\left[5 \times 10^{3}-8 \times 10^{4}\right] \mathrm{cm}^{-3} \mathrm{~K}$ (Joblin et al. 2018; Wu et al. 2018). Interestingly, this correlation is predicted, almost independent of the initial gas density, by non-stationary hydrodynamical models of photoevaporative PDRs (Bron et al. 2018). The high pressures inferred at the PDR surface, often unbalanced by those of the surrounding environment, would then have a dynamical origin: cloud edge heating, compression, and photoevaporation. These proceses

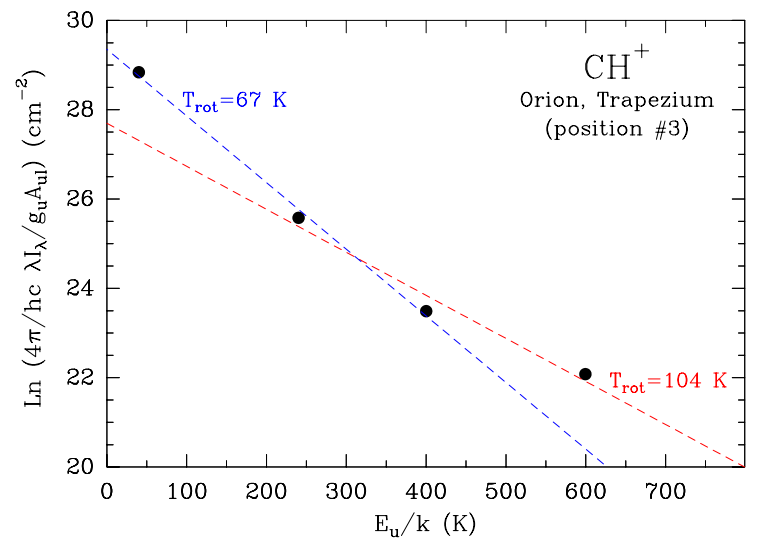

Fig. 9. $\mathrm{CH}^{+}$rotational population diagram $\left(J_{\mathrm{u}}=1-5\right)$ obtained from Herschel/PACS and HIFI observations toward position\#3 near the Trapezium region. The straight lines and associated rotational temperatures are fits to the emission lines in different $J$ ranges.

greatly depend on the strength and shape of the stellar UV radiation field (Hill \& Hollenbach 1978; Bertoldi 1989; Bertoldi \& Draine 1996; Störzer \& Hollenbach 1998; Hosokawa \& Inutsuka 2006; Pellegrini et al. 2009; Bron et al. 2018). ALMA observations of the Orion Bar PDR do suggest the presence of photoevaporative flows of neutral gas (Goicoechea et al. 2016).

\subsection{PDR modeling}

We have used the Meudon PDR code (e.g., Le Petit et al. 2006; Goicoechea \& Le Bourlot 2007; Bron et al. 2014) to model 


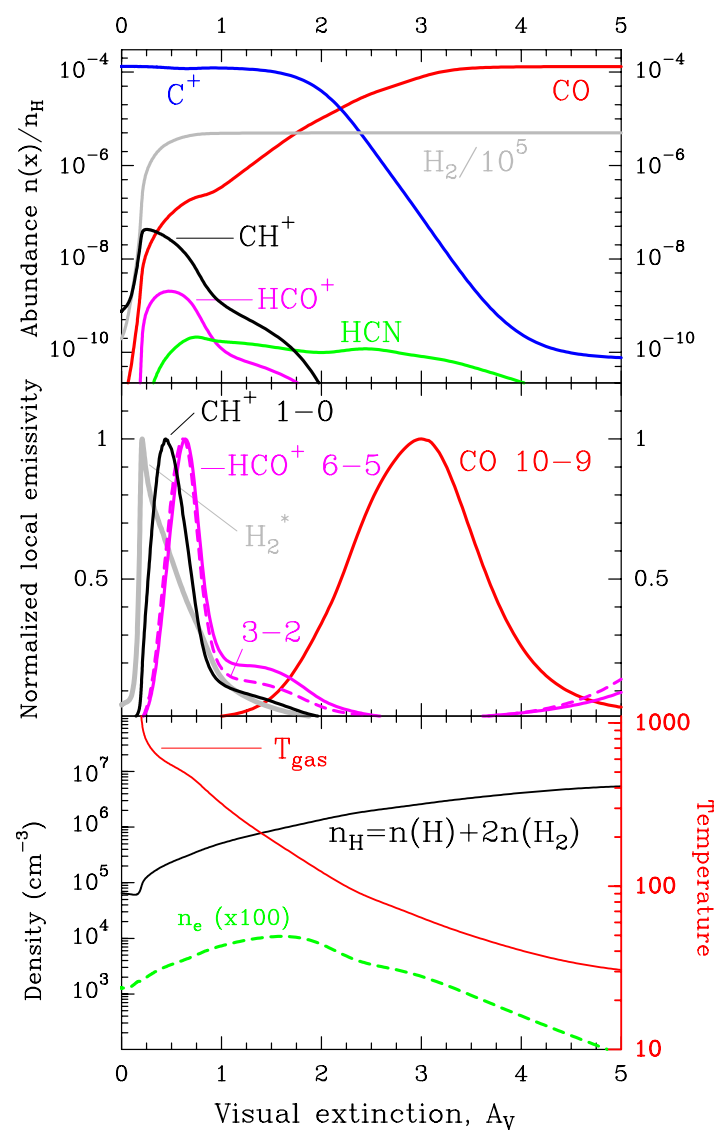

Fig. 10. Isobaric PDR model with $P_{\text {th }}=10^{8} \mathrm{~cm}^{-3} \mathrm{~K}$ and $G_{0}=7.5 \times 10^{3}$, the mean $G_{0}$ in OMC-1. Top panel: fractional abundances as a function of visual extinction $\left(A_{\mathrm{V}}\right)$ into the molecular cloud. Middle panel: normalized local line emissivities. $\mathrm{H}_{2}^{*}$ stands for the $\mathrm{H}_{2} v=1-0 S(1)$ line at $2.12 \mu \mathrm{m}$. Bottom panel: predicted gas temperature $\left(T_{\text {gas }}\right)$, hydrogen nuclei gas density $\left(n_{\mathrm{H}}\right)$ and electron density $\left(n_{\mathrm{e}}\right)$ gradients.

the cloud edge layers where the observed submm line emission arises. The model simulates a stationary PDR; the penetration of FUV photons, the thermal balance, and the chemistry are computed self-consistently. We adopted an extinction to color-index ratio, $R_{\mathrm{V}}=A_{\mathrm{V}} / E_{\mathrm{B}-\mathrm{V}}$, of 5.5 consistent with the flatter extinction curve toward Orion (Cardelli et al. 1989). Regarding $\mathrm{CH}^{+}$ formation, these models include an $\mathrm{H}_{2}$ state-dependent treatment of Eq. (1) (see Agúndez et al. 2010; Zanchet et al. 2013; Herráez-Aguilar et al. 2014; Faure et al. 2017). In particular, the $\mathrm{CH}^{+}$formation rate is computed by summing over all formation rates for each specific state of $\mathrm{H}_{2}$.

Bron et al. (2018) concluded that, in the frame of stationary PDR models, a better description of the PDR compressed layers, typically between $A_{\mathrm{V}}=0$ and $\sim 5$ mag into the cloud, is obtained by constant pressure models instead of the constant density models often used in the literature. With this in mind, in Fig. 10 we show a representative stationary PDR model adapted to the irradiated edge of OMC-1. This is a high-pressure, $P_{\text {th }}=10^{8} \mathrm{~cm}^{-3} \mathrm{~K}$, isobaric PDR illuminated by the mean FUV radiation flux in the region mapped by HIFI $\left(\left\langle G_{0}\right\rangle \simeq 7.5 \times 10^{3}\right)$. The bottom panel in Fig. 10 shows the predicted physical structure, from $A_{\mathrm{V}}=0$ to 5 mag of visual extinction into the molecular cloud. The upper panel shows fractional abundances of $\mathrm{H}_{2}, \mathrm{C}^{+}, \mathrm{CH}^{+}, \mathrm{CO}, \mathrm{HCO}^{+}$ and $\mathrm{HCN}$ with respect to $\mathrm{H}$ nuclei. We note that $\mathrm{CH}^{+}$and $\mathrm{HCO}^{+}$ abundances peak at the edge of the PDR, before the $\mathrm{C}^{+}$to $\mathrm{CO}$ transition layers, that is, closer to the H II region. Therefore,

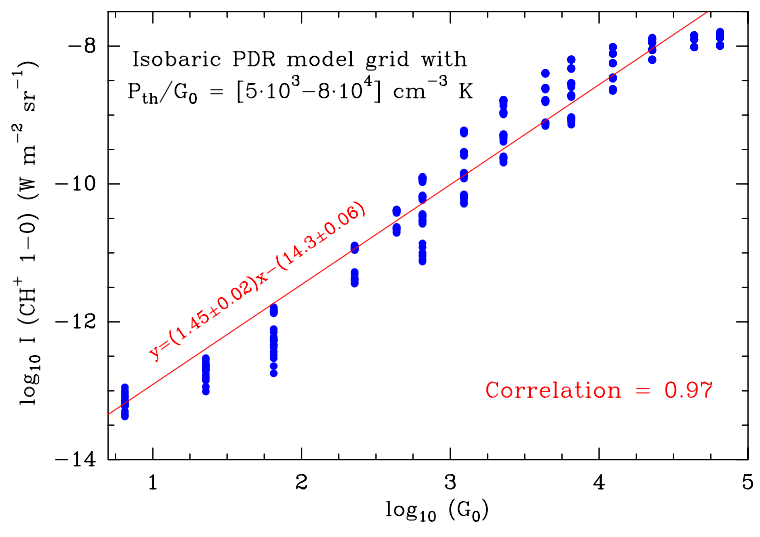

Fig. 11. Results from a grid of isobaric PDR models with the $P_{\mathrm{th}} / G_{0}$ ratio ranging from $5 \times 10^{3}$ to $8 \times 10^{4} \mathrm{~cm}^{-3} \mathrm{~K}$ (see text). The plot shows a correlation between incident FUV flux, $G_{0}$, and $\mathrm{CH}^{+}(J=1-0)$ intensity.

both $\mathrm{CH}^{+}$and $\mathrm{HCO}^{+}$are abundant ${ }^{4}$ in layers where the ionization fraction, driven by the photoionization of carbon atoms, is high, $x\left(e^{-}\right) \approx 10^{-4}$. The high electron density in these layers, $n_{e} \simeq 30-100 \mathrm{~cm}^{-3}$, contributes to the collisional excitation of their rotational levels. The middle panel in Fig. 10 shows the predicted local emissivities of the observed molecular lines, normalized by their emission peak. In the optically thin limit, the actual line intensities will follow the same spatial distribution.

Because of the efficient chemical formation of $\mathrm{HCO}^{+}$ from reactive ions $\mathrm{CH}^{+}$and $\mathrm{CO}^{+}$at the PDR surface, before the $\mathrm{C}^{+}$to $\mathrm{CO}$ transition (e.g., Sternberg \& Dalgarno 1995; Goicoechea et al. 2016), the $\mathrm{CH}^{+} J=1-0$ and $\mathrm{HCO}^{+} J=6-5$ lines are predicted to trace hotter gas $\left(T_{\mathrm{k}} \simeq 500 \mathrm{~K}\right.$ in this model), than the CO $J=10-9$ emission. The CO $J=10-9$ line, traces cooler and an order of magnitude denser gas, $n_{\mathrm{H}} \simeq 10^{6} \mathrm{~cm}^{-3}$, that arises from slightly deeper inside the PDR. Given the relatively high gas densities in these warm PDR layers, the observed submm molecular lines arise from a very thin layer: $\sim 1.6 \times 10^{16} \mathrm{~cm}^{-2} \simeq 5 \times 10^{-3} \mathrm{pc} \simeq 1000 \mathrm{AU}$ in this particular model. This is not far from previous claims, based on lowangular resolution observations, for the dense molecular gas confining the H II region M42 (e.g., Rodríguez-Franco et al. 2001). At the distance to Orion, these spatial scales imply angular thicknesses of only several arcseconds. In other words, they cannot be spatially resolved by single-dish telescopes. This explains the similar morphology of the $\mathrm{CH}^{+} J=1-0$ and $\mathrm{CO} J=10-9$ extended emission observed by Herschel. ALMA images of $\mathrm{SH}^{+}$and $\mathrm{HCO}^{+}(J=4-3)$ emission do resolve the compressed, warm layers of the Orion Bar PDR (Goicoechea et al. 2016, 2017).

We also analyzed the results of a grid of $\sim 1300$ stationary, isobaric PDR models, also run with the Meudon code, and searched for a theoretical validation of the observed $I\left(\mathrm{CH}^{+} 1-0\right)$ versus $G_{0}$ correlation (Sect. 4.3). Figure 11 shows results from models that cover a wide parameter space in $G_{0}$ and $P_{\text {th }}$, and satisfy the condition $P_{\mathrm{th}} / G_{0}$ from $5 \times 10^{3}$ to $8 \times 10^{4} \mathrm{~cm}^{-3} \mathrm{~K}$, the same range obtained from photoevaporative PDR models (Bron et al. 2018) and also suggested by observations (e.g., Joblin et al. 2018; Wu et al. 2018). In this broad parameter space of illumination conditions, models do predict a correlation between incident FUV flux and $\mathrm{CH}^{+} J=1-0$ line intensity.

${ }_{4} \mathrm{HCO}^{+}$is also abundant deep inside the shielded molecular cloud, mainly formed by the reaction $\mathrm{CO}+\mathrm{H}_{3}^{+} \rightarrow \mathrm{HCO}^{+}+\mathrm{H}_{2}$. 
The slope of the predicted correlation, in logarithmic scale, is $m_{\text {mod }}=1.45 \pm 0.02$ (Fig. 11). This value is only slightly above the one inferred from our observations of OMC- $1, m_{\mathrm{obs}}=1.0 \pm 0.1$, but covering regions of higher FUV fluxes, $G_{0} \gtrsim 10^{3}$. This satisfactory agreement suggests that, for a wide range of $G_{0}$ values, the stationary isobaric PDR model does capture the global properties of the compressed PDR layers at the irradiated edges of OMC-1. It also supports the observational result that the $\mathrm{CH}^{+}$ $J=1-0$ emission traces the strength of the incident FUV flux.

\section{2. $\mathrm{CH}^{+}$, an unambiguous tracer of FUV-irradiated gas}

The [C II] $158 \mu \mathrm{m}$ line emission is the most luminous tracer of the extended FUV-irradiated neutral gas in the Milky Way (e.g., Bennett et al. 1994). In some circumstances, however, the interpretation of [C II] $158 \mu \mathrm{m}$ observations is not trivial: uncertain contribution from $\mathrm{H}$ II ionized gas, multiple velocity components along a given line-of-sight, optical depth effects toward bright and dense PDRs, etc. (see, e.g., Ossenkopf et al. 2013; Pabst et al. 2017). The detection of rotational emission from reactive molecular ions such as $\mathrm{CH}^{+}$or $\mathrm{SH}^{+}$is an alternative and unambiguous signature of FUV-irradiated dense ${ }^{5}$ molecular gas (Black 1998; Nagy et al. 2013; Goicoechea et al. 2017). We have shown that the intensity of the $\mathrm{CH}^{+} J=1-0$ line scales with $G_{0}$. Despite the elevated endothermicity of reaction $\mathrm{C}^{+}+\mathrm{H}_{2}(v=0) \rightarrow$ $\mathrm{CH}^{+}+\mathrm{H}$, a high FUV photon flux enhances the gas temperature, the column density of FUV-pumped $\mathrm{H}_{2}(v \geq 1)$, and that of $\mathrm{CH}^{+}$ (Sternberg \& Dalgarno 1995; Agúndez et al. 2010; Faure et al. 2017). We conclude that reaction (1) is not only efficient locally, in bright and dense PDR/H II interfaces such as the Bar. It is also efficient at large spatial scales where $\mathrm{CH}^{+}(J=1-0)$ traces an extended but thin layer of FUV-irradiated molecular gas.

\subsection{Observational tracers of the radiative feedback from massive stars and extragalactic link}

Compared to the lowest-energy " $J=1-0$ " rotational lines of $\mathrm{CO}$, $\mathrm{HCN}, \mathrm{HCO}^{+}$, or $\mathrm{N}_{2} \mathrm{H}^{+}$typically mapped from ground-based telescopes (e.g., Pety et al. 2017; Hacar et al. 2017; Kauffmann et al. 2017), the more excited submm "mid- $J$ " lines are better diagnostics of the high-pressure gas in star-forming regions (SFRs). Because FUV radiation and shocks dominate the heating of the warm gas in molecular clouds, mid- $J$ lines are more sensitive probes of the radiative and mechanical feedback from stars and protostars. This warm gas component can dominate the line luminosity emitted by SFRs in the Milky Way, and from distant star-forming galaxies as a whole.

Most spectroscopic observations carried out by Herschel toward nearby SFRs (e.g., van Dishoeck et al. 2011) targeted low-mass protostars and their outflows (e.g., Nisini et al. 2010; Kristensen et al. 2012; Herczeg et al. 2012; Goicoechea et al. 2012; Karska et al. 2013; Manoj et al. 2013), as well as more distant high-mass star-forming cores (e.g., Bergin et al. 2010; Etxaluze et al. 2013; van der Tak et al. 2013; Karska et al. 2014; Goicoechea et al. 2015b; Indriolo et al. 2017). These are relatively compact sources, at Herschel's angular resolution, producing intense FIR high- $J \mathrm{CO}$ and $\mathrm{H}_{2} \mathrm{O}$ line emission that arises from hot molecular gas, $T_{\text {rot }}(\mathrm{CO}) \gtrsim 300 \mathrm{~K}$, associated to protostellar outflows. The observed $\mathrm{CO}$ rotational emission ladder toward these sources typically peaks at $J_{\text {peak }}>15$ and shows detectable $\mathrm{CO}$ emission at $J \gtrsim 30$. Although at the spatial scales

\footnotetext{
5 We refer to dense molecular gas, $n_{\mathrm{H}} \gtrsim 10^{5} \mathrm{~cm}^{-3}$, as opposed to the detection of $\mathrm{CH}^{+}(J=1-0)$ absorption from low-density, $n_{\mathrm{H}} \lesssim 100 \mathrm{~cm}^{-3}$, diffuse interstellar clouds (e.g., Godard et al. 2012).
}

of an entire GMC, the filling factor of these protostellar outflows is small, the shape of their CO spectral line energy distribution (SLED) is representative of hot, shock-heated molecular gas. In a broader context, this shock-heated component may dominate the observed FIR high- $J \mathrm{CO}$ emission from active galactic nuclei (AGN) galaxies in which these lines are readily detected (e.g., Sturm et al. 2010; Hailey-Dunsheath et al. 2012).

The CO line emission measured by Herschel toward less extreme star-forming galaxies typically peaks at $J_{\text {peak }} \lesssim 10$ (e.g., Kamenetzky et al. 2012, 2014; Indriolo et al. 2017). Hence, most of the mid- $J$ CO luminosity emitted at kpc scales likely arises from non-shocked warm molecular gas. In normal (Milky Way type) and starburst galaxies, the observed mid- $J$ emission possibly arises from widespread PDR gas, similar to that in the extended irradiated surface of OMC-1, that is, relatively dense molecular gas exposed to FUV radiation from OB-type stars. As in any local PDR, this warm gas component copiously emits at FIR and submm wavelengths (Hollenbach \& Tielens 1997). This emission includes bright CO lines with a SLED peaking at $J_{\text {peak }} \simeq 10-15$ for strongly irradiated Orion Bar-like PDRs with $G_{0}>10^{3}$ (Joblin et al. 2018), at $J_{\text {peak }} \gtrsim 7-6$ for lower FUV-radiation fields, $G_{0}<10^{3}$, such as the extended envelope around Sgr B2 cloud (Etxaluze et al. 2013), or the Horsehead PDR, with $G_{0} \simeq 10^{2}$.

Using OMC-1 as template to quantify the radiative impact of young massive stars on their natal cloud, we see that the FUV flux is still strong enough to drive the gas heating and chemistry of an extended gas component of warm, $T_{\mathrm{k}} \approx 100-150 \mathrm{~K}$, molecular gas at pc distances from massive stars. This is seen from the larger extent of the CO $J=10-9$ emission compared to that of $\mathrm{C}^{18} \mathrm{O} J=2-1$ (Fig. 2). The $\mathrm{HCO}^{+}$and $\mathrm{HCN} J=6-5$ maps reveal that the gas density at the cloud edges are relatively high, $n_{\mathrm{H}} \gtrsim 10^{5}-10^{6} \mathrm{~cm}^{-3}$, so the thermal pressures are high. Such densities may be related to a particularly dense condensation that gave rise to the Trapezium stars; they may be related to the cloud evolution itself, as a consequence of the global gravitational collapse of OMC-1 (e.g., Hartmann \& Burkert 2007; Hacar et al. 2017); or produced by the FUV-induced compression of the cloud surfaces (e.g., Goicoechea et al. 2016; Bron et al. 2018).

Square-degree maps of the GMCs Orion A and B in the HCN $J=1-0$ line emission have revealed that, despite the high critical density of this transition, several $10^{6} \mathrm{~cm}^{-3}$, most of the emission arises from relatively low-density gas $\left(n_{\mathrm{H}} \approx 10^{3} \mathrm{~cm}^{-3}\right.$; Pety et al. 2017; Kauffmann et al. 2017). Therefore, the mere observation of a low-energy but high critical density line does not immediately imply that the emission arises from cold and dense gas, the fuel that ultimately forms stars, as often assumed in the interpretation of extragalactic observations (e.g., Gao \& Solomon 2004; Usero et al. 2015). The very extended HCN $(J=1-0)$ emission detected along GMCs of the Milky Way just reveals emission from weakly collisionally-excited gas $\left(T_{\text {ex }}(1-0) \ll T_{\mathrm{k}}\right)$, perhaps assisted by electron excitation if the ionization fraction is high enough due to FUV irradiation (e.g., Goldsmith \& Kauffmann 2017). This may be the case of many galaxies too. On the other hand, our detection of extended HCN $J=6-5$ emission and of large $\mathrm{HCO}^{+} 6-5 / 3-2$ intensity ratios in $\mathrm{OMC}-1$ does demonstrate the presence warm and dense 5 molecular gas at $1 \mathrm{pc}^{2}$ scales.

The $\mathrm{HCN}$ to $\mathrm{HCO}^{+} J=6-5$ line ratio $(R)$ map of OMC-1 shown in Fig. B.4 further differentiates the extended gas illuminated by FUV radiation $(R<0.5)$, from the very high IR-luminosity regions, such as $\mathrm{BN} / \mathrm{KL}$ with $R \simeq 2$. These are regions hosting on-going massive star formation and powerful 
protostellar outflows still buried in large column densities of FIRemitting dust. In the extragalactic context, ultraluminous infrared galaxies (ULIRGs), that is, merger galaxies characterized by very high star formation rates and massive nuclear outflows (e.g., González-Alfonso et al. 2017, and references therein) also display elevated $R \simeq 1-2$ luminosity ratios (e.g., Krips et al. 2008; Imanishi et al. 2018, and references therein). Pure starburst galaxies, however, show $R$ ratios smaller than one (e.g., Salas et al. 2014; Aladro et al. 2015), similar to our observed value for the extended gas in OMC-1.

The recent ALMA detection of $\mathrm{CH}^{+}(J=1-0)$ emission and absorption toward ULIRGs at $z \sim 2$ (Falgarone et al. 2017) demonstrates the surprisingly widespread nature of $\mathrm{CH}^{+}$rotational emission, also in distant and extreme galaxies. The absorbing $\mathrm{CH}^{+}$presumably arises from a turbulent diffuse gas reservoir. The emission component, emitted at kiloparsec scales, is interpreted as denser gas affected by a collection of multiple FUV-irradiated shocks (needed to explain the broad-line profiles) individually propagating at velocities of $v_{\text {shock }} \sim 40 \mathrm{~km} \mathrm{~s}^{-1}$, and driven by galactic winds (Falgarone et al. 2017). Our detection of parsec scale $\mathrm{CH}^{+}(J=1-0)$ emission toward OMC-1, a star-forming cloud core irradiated by FUV photons from just a few young massive stars, provides an example of extended $\mathrm{CH}^{+}$ emission from non-shocked gas $\left(\Delta \mathrm{v} \sim 5 \mathrm{~km} \mathrm{~s}^{-1}\right)$. Because the lifetime of $\mathrm{CH}^{+}$in dense gas is so short, this $\mathrm{CH}^{+}$emission traces the instantaneous feedback from massive stars. This may be the case of many starburst galaxies producing detectable $\mathrm{CH}^{+}$ rotational emission too.

\section{Summary and conclusions}

We have presented $\sim 85 \operatorname{arcmin}^{2}(\sim 0.9 \mathrm{pc} \times 1.4 \mathrm{pc})$ velocityresolved maps of the $\mathrm{CH}^{+}(J=1-0), \mathrm{CO}(J=10-9), \mathrm{H}_{2} \mathrm{O}$ $\left(3_{12}-22_{21}\right), \mathrm{CH}_{3} \mathrm{OH}, \mathrm{CH}(N, J=1,3 / 2-1,1 / 2)$, HCN $(J=6-5$ and 13-12), and $\mathrm{HCO}^{+}(J=6-5)$ lines, obtained with the heterodyne instrument Herschel/HIFI toward the closest high-mass star-forming region, OMC-1. In combination with archival NIR photometric images tracing the $\mathrm{H}_{2}(v \geq 1)$ emission, and new $\mathrm{HCO}^{+} J=3-2$ and $\mathrm{C}^{18} \mathrm{O} J=2-1$ line maps taken with the IRAM $30 \mathrm{~m}$ telescope, we obtained the following results:

- The rotational emission from reactive ion $\mathrm{CH}^{+}$is very extended. The $\mathrm{CH}^{+}(J=1-0)$ emission spatially correlates with that of [C II] $158 \mu \mathrm{m}$ and of FUV-pumped $\mathrm{H}_{2}$. The observed correlations imply that the reaction of $\mathrm{C}^{+}$ions with $\mathrm{H}_{2}(v \geq 1)$ molecules dominates the formation of $\mathrm{CH}^{+}$, not only locally toward dense PDR/H II interfaces, but also at parsec scales.

- The extended $\mathrm{CO}(J=10-9)$ and $\mathrm{HCO}^{+}$(mid- $J$ ) narrowline emission $\left(\Delta v \simeq 3 \mathrm{~km} \mathrm{~s}^{-1}\right)$ traces a thin layer of warm gas at the surface of the molecular cloud $\left(A_{\mathrm{V}} \simeq 3-6\right.$ mag or $\sim 10^{16} \mathrm{~cm}$ in thickness) illuminated by FUV radiation from massive stars in the Trapezium cluster. This layer, with a mass density of $120-240 M_{\odot} \mathrm{pc}^{-2}$, accounts for $\sim 5-10 \%$ of the total gas mass in OMC-1. Using non-LTE excitation models we infer high thermal pressures, $P_{\mathrm{th}}=T_{k} \cdot n_{\mathrm{H}} \gtrsim 10^{7}-10^{9} \mathrm{~cm}^{-3} \mathrm{~K}$, for this gas component. This is consistent with the expected dynamical response of molecular clouds to strong FUV radiation: the cloud edge is heated and compressed, and photoevaporates if the high-pressures in the PDR are not balanced by those of the environment (e.g., Bertoldi \& Draine 1996; Bron et al. 2018).

- Photoevaporative PDR models predict that the quasiconstant thermal pressure in the compressed PDR layers scales with the strength of the FUV photon flux impinging the cloud (Bron et al. 2018). $\mathrm{CH}^{+}$proves to be a unique tracer of these narrow layers. In this work we have found a spatial correlation between the intensity of the $\mathrm{CH}^{+}(J=1-0)$ line and $G_{0}$, ranging from $G_{0} \sim 10^{3}-10^{5}$ in OMC- 1 . The observed correlation is supported, and enlarged to lower $G_{0}$ values, by isobaric models in the parameter space $P_{\mathrm{th}} / G_{0} \approx\left[5 \times 10^{3}-8 \times 10^{4}\right] \mathrm{cm}^{-3} \mathrm{~K}$ where many PDRs seem to lie (Joblin et al. 2018; Wu et al. 2018). This correlation also implies that for moderate gas densities, $n_{\mathrm{H}}>10^{5} \mathrm{~cm}^{-3}$, the $\mathrm{CH}^{+}(J=1-0) /[\mathrm{C} \mathrm{II}] 158 \mu \mathrm{m}$ intensity ratio traces gas density variations at the irradiated cloud surfaces.

- We detect bright and rotationally warm, $T_{\text {rot }} \simeq 100 \mathrm{~K}$, $\mathrm{CH}^{+}$emission toward the most irradiated regions facing the Trapezium cluster. The detection of excited $\mathrm{CH}^{+}$lines, up to $J=5-4$, is consistent with its exothermic formation from $\mathrm{H}_{2}(v \geq 1)$, and with the formation pumping mechanism that enhances the population of $\mathrm{CH}^{+}$rotationally excited levels (Godard \& Cernicharo 2013; Faure et al. 2017). The broad $J=1-0$ linewidths are consistent with the high reactivity of the molecule: $\mathrm{CH}^{+}$can be excited by radiation many times during its short lifetime (a few hours), and during its mean-free-time for elastic and inelastic collisions, so that it remains kinetically hot (large velocity dispersion) and rotationally warm (high $T_{\text {rot }}$ ) while it emits (e.g., Black 1998; Goicoechea et al. 2017).

The detection of extended, parsec-scale $\mathrm{CH}^{+}(J=1-0)$ and narrow-line mid- $J$ CO emission in OMC-1, both lines arising from PDR gas and not from fast shocks, probes the radiative interaction between young massive stars and their natal molecular cloud. This radiative feedback alters the dynamics, physical conditions, and chemistry of the most exposed neutral cloud layers. In turn, although not the most massive cloud core component, these PDR layers dominate the line luminosity emitted by GMCs. Similar processes must take place in other clusters hosting more numerous and more massive stars (e.g., Wareing et al. 2018). They likely occur in starburst galaxies as well. There, the PDR line emission from $\mathrm{C}^{+}$, mid- $J \mathrm{CO}$, and reactive ions such as $\mathrm{CH}^{+}$must reach larger spatial scales, and can dominate the emitted line luminosity too.

Acknowledgements. We thank the Spanish MICIU for funding support under grants AYA2016-75066-C2-1-P and AYA2017-85111-P, and the ERC for support under grant ERC-2013-Syg-610256-NANOCOSMOS.

\section{References}

Agúndez, M., Goicoechea, J. R., Cernicharo, J., Faure, A., \& Roueff, E. 2010, ApJ, 713, 662

Aladro, R., Martín, S., Riquelme, D., et al. 2015, A\&A, 579, A101

Arab, H., Abergel, A., Habart, E., et al. 2012, A\&A, 541, A19

Bally, J. 2008, in Handbook of Star Forming Regions, ed. B. Reipurth (San Francisco, CA: Astronomical Society of the Pacific), 459

Bally, J., Langer, W. D., Stark, A. A., \& Wilson, R. W. 1987, ApJ, 312, L45

Bally, J., Cunningham, N. J., Moeckel, N., et al. 2011, ApJ, 727, 113

Becklin, E. E., \& Neugebauer, G. 1967, ApJ, 147, 799

Beckwith, S., Persson, S. E., Neugebauer, G., \& Becklin, E. E. 1978, ApJ, 223, 464

Bennett, C. L., Fixsen, D. J., Hinshaw, G., et al. 1994, ApJ, 434, 587

Benz, A. O., Bruderer, S., van Dishoeck, E. F., et al. 2010, A\&A, 521, L35

Benz, A. O., Bruderer, S., van Dishoeck, E. F., et al. 2016, A\&A, 590, A105

Bergin, E. A., Phillips, T. G., Comito, C., et al. 2010, A\&A, 521, L20

Berné, O., Marcelino, N., \& Cernicharo, J. 2014, ApJ, 795, 13

Bertoldi, F. 1989, ApJ, 346, 735

Bertoldi, F., \& Draine, B. T. 1996, ApJ, 458, 222

Beuther, H., Churchwell, E. B., McKee, C. F., \& Tan, J. C. 2007, in Protostars and Planets V, eds. B. Reipurth, D. Jewitt, \& K. Keil (Tucson, AZ: University of Arizona Press), 165

Black, J. H. 1998, Faraday Discuss., 109, 257

Blake, G. A., Sutton, E. C., Masson, C. R., \& Phillips, T. G. 1987, ApJ, 315, 621 Bron, E., Le Bourlot, J., \& Le Petit F. 2014, A\&A, 569, A100

Bron, E., Agúndez, M., Goicoechea, J. R., \& Cernicharo, J. 2018, A\&A, submitted [arXiv:1801.01547]

Burton, M. G., Hollenbach, D. J., \& Tielens, A. G. G. M. 1990, ApJ, 365, 620 
Cardelli, J. A., Clayton, G. C., \& Mathis, J. S. 1989, ApJ, 345, 245

Carroll, T. J., \& Goldsmith, P. F. 1981, ApJ, 245, 891

Cernicharo, J., Liu, X.-W., González-Alfonso, E., et al. 1997, ApJ, 483, L65

Chen, J.-H., Goldsmith, P. F., Viti, S., et al. 2014, ApJ, 793, 111

Cuadrado, S., Goicoechea, J. R., Pilleri, P., et al. 2015, A\&A, 575, A82

Cuadrado, S., Goicoechea, J. R., Roncero, O., et al. 2016, A\&A, 596, L1

Cuadrado, S., Goicoechea, J. R., Cernicharo, J., et al. 2017, A\&A, 603, A124

Dalgarno, A., \& McCray, R. A. 1972, ARA\&A, 10, 375

de Graauw, T., Helmich, F. P., Phillips, T. G., et al. 2010, A\&A, 518, L6

Douglas, A. E., \& Herzberg, G. 1941, ApJ, 94, 381

Draine, B. T. 1995, Ap\&SS, 233, 111

Draine, B. T., \& Katz, N. 1986, ApJ, 310, 392

Elitzur, M., \& Watson, W. D. 1978, ApJ, 222, L141

Etxaluze, M., Goicoechea, J. R., Cernicharo, J., et al. 2013, A\&A, 556, A137

Falgarone, E., Pineau des Forets, G., \& Roueff, E. 1995, A\&A, 300, 870

Falgarone, E., Godard, B., Cernicharo, J., et al. 2010a, A\&A, 521, L15

Falgarone, E., Ossenkopf, V., Gerin, M., et al. 2010b, A\&A, 518, L118

Falgarone, E., Zwaan, M. A., Godard, B., et al. 2017, Nature, 548, 430

Faure, A., \& Tennyson, J. 2001, MNRAS, 325, 443

Faure, A., Halvick, P., Stoecklin, T., et al. 2017, MNRAS, 469, 612

Flower, D. R. 1999, MNRAS, 305, 651

Fuente, A., Rodrıguez-Franco, A., Garcia-Burillo, S., Martın-Pintado, J., \& Black, J. H. 2003, A\&A, 406, 899

Fuente, A., García-Burillo, S., Usero, A., et al. 2008, A\&A, 492, 675

Gao, Y., \& Solomon, P. M. 2004, ApJ, 606, 271

Genzel, R., \& Stutzki, J. 1989, ARA\&A, 27, 41

Gerin, M., Ruaud, M., Goicoechea, J. R., et al. 2015, A\&A, 573, A30

Gerin, M., Neufeld, D. A., \& Goicoechea, J. R. 2016, ARA\&A, 54, 181

Gibb, E. L., Whittet, D. C. B., Boogert, A. C. A., \& Tielens, A. G. G. M. 2004, ApJS, 151, 35

Godard, B., \& Cernicharo, J. 2013, A\&A, 550, A8

Godard, B., Falgarone, E., \& Pineau Des Forêts G. 2009, A\&A, 495, 847

Godard, B., Falgarone, E., Gerin, M., et al. 2012, A\&A, 540, A87

Godard, B., Falgarone, E., \& Pineau des Forêts, G. 2014, A\&A, 570, A27

Goicoechea, J. R., \& Le Bourlot J. 2007, A\&A, 467, 1

Goicoechea, J. R., Pety, J., Gerin, M., et al. 2006, A\&A, 456, 565

Goicoechea, J. R., Cernicharo, J., Karska, A., et al. 2012, A\&A, 548, A77

Goicoechea, J. R., Teyssier, D., Etxaluze, M., et al. 2015a, ApJ, 812, 75

Goicoechea, J. R., Chavarría, L., Cernicharo, J., et al. 2015b, ApJ, 799, 102

Goicoechea, J. R., Pety, J., Cuadrado, S., et al. 2016, Nature, 537, 207

Goicoechea, J. R., Cuadrado, S., Pety, J., et al. 2017, A\&A, 601, L9

Goldsmith, P. F., \& Kauffmann, J. 2017, ApJ, 841, 25

Goldsmith, P. F., Bergin, E. A., \& Lis, D. C. 1997, ApJ, 491, 615

Goldsmith, P. F., Langer, W. D., Pineda, J. L., \& Velusamy, T. 2012, ApJS, 203, 13

González-Alfonso, E., Wright, C. M., Cernicharo, J., et al. 2002, A\&A, 386, 1074

González-Alfonso, E., Fischer, J., Spoon, H. W. W., et al. 2017, ApJ, 836, 11

GRAVITY Collaboration (Karl, M., et al.) 2018, A\&A, 620, A116

Großschedl, J. E., Alves, J., Meingast, S., et al. 2018, A\&A, 619, A106

Habing, H. J. 1968, Bull. Astron. Inst. Netherlands, 19, 421

Hacar, A., Alves, J., Tafalla, M., \& Goicoechea, J. R. 2017, A\&A, 602, L2

Hacar, A., Tafalla, M., Forbrich, J., et al. 2018, A\&A, 610, A77

Haid, S., Walch, S., Seifried, D., et al. 2018, MNRAS, 478, 4799

Hailey-Dunsheath, S., Sturm, E., Fischer, J., et al. 2012, ApJ, 755, 57

Hartmann, L., \& Burkert, A. 2007, ApJ, 654, 988

Herczeg, G. J., Karska, A., Bruderer, S., et al. 2012, A\&A, 540, A84

Herráez-Aguilar, D., Jambrina, P. G., Menéndez, M., et al. 2014, Phys. Chem. Chem. Phys., 16, 24800

Herrmann, F., Madden, S. C., Nikola, T., et al. 1997, ApJ, 481, 343

Hierl, P. M., Morris, R. A., \& Viggiano, A. A. 1997, J. Chem. Phys., 106, 10145

Hill, J. K., \& Hollenbach, D. J. 1978, ApJ, 225, 390

Hogerheijde, M. R., Jansen, D. J., \& van Dishoeck E. F. 1995, A\&A, 294, 792

Hollenbach, D. J., \& Tielens, A. G. G. M. 1997, ARA\&A, 35, 179

Hosokawa, T., \& Inutsuka, S.-i. 2006, ApJ, 646, 240

Imanishi, M., Nakanishi, K., \& Izumi, T. 2018, ApJ, 856, 143

Indriolo, N., Bergin, E. A., Goicoechea, J. R., et al. 2017, ApJ, 836, 117

Jiménez-Serra, I., Caselli, P., Martín-Pintado, J., \& Hartquist, T. W. 2008, A\&A, 482,549

Joblin, C., Bron, E., Pinto, C., et al. 2018, A\&A, 615, A129

Joulain, K., Falgarone, E., Pineau des Forets, G., \& Flower, D. 1998, A\&A, 340,

Kamenetzky, J., Glenn, J., Rangwala, N., et al. 2012, ApJ, 753, 70

Kamenetzky, J., Rangwala, N., Glenn, J., Maloney, P. R., \& Conley, A. 2014, ApJ, 795, 174

Kaplan, K. F., Dinerstein, H. L., Oh, H., et al. 2017, ApJ, 838, 152

Karska, A., Herczeg, G. J., van Dishoeck, E. F., et al. 2013, A\&A, 552, A141

Karska, A., Herpin, F., Bruderer, S., et al. 2014, A\&A, 562, A45
Kaufman, M. J., Wolfire, M. G., Hollenbach, D. J., \& Luhman, M. L. 1999, ApJ, 527,795

Kauffmann, J., Goldsmith, P. F., Melnick, G., et al. 2017, A\&A, 605, L5

Krips, M., Neri, R., García-Burillo, S., et al. 2008, ApJ, 677, 262

Kristensen, L. E., van Dishoeck, E. F., Bergin, E. A., et al. 2012, A\&A, 542, A8

Krumholz, M. R., Bate, M. R., Arce, H. G., et al. 2014, in Protostars and Planets

VI, eds. H. Beuther, et al. (Tucson, AZ: University of Arizona Press), 243

Kwan, J., \& Scoville, N. 1976, ApJ, 210, L39

Langer, W. D., \& Penzias, A. A. 1990, ApJ, 357, 477

Le Petit, F., Nehmé, C., Le Bourlot, J., \& Roueff, E. 2006, ApJS, 164, 506

Lesaffre, P., Gerin, M., \& Hennebelle, P. 2007, A\&A, 469, 949

Luhman, M. L., Jaffe, D. T., Keller, L. D., \& Pak, S. 1994, ApJ, 436, L185

Manoj, P., Watson, D. M., Neufeld, D. A., et al. 2013, ApJ, 763, 83

McKee, C. F., \& Ostriker, E. C. 2007, ARA\&A, 45, 565

Meingast, S., Alves, J., Mardones, D., et al. 2016, A\&A, 587, A153

Melnick, G. J., \& Kaufman, M. J. 2015, ApJ, 806, 227

Melnick, G. J., Tolls, V., Neufeld, D. A., et al. 2010, A\&A, 521, L27

Menten, K. M., Reid, M. J., Forbrich, J., \& Brunthaler, A. 2007, A\&A, 474, 515

Morris, P. W., Gupta, H., Nagy, Z., et al. 2016, ApJ, 829, 15

Nagy, Z., Van der Tak, F. F. S., Ossenkopf, V., et al. 2013, A\&A, 550, A96

Nagy, Z., Choi, Y., Ossenkopf-Okada, V., et al. 2017, A\&A, 599, A22

Naylor, D. A., Dartois, E., Habart, E., et al. 2010, A\&A, 518, L117

Nisini, B., Benedettini, M., Codella, C., et al. 2010, A\&A, 518, L120

O'Dell, C. R. 2001, ARA\&A, 39, 99

O’Dell, C. R., Henney, W. J., Abel, N. P., Ferland, G. J., \& Arthur, S. J. 2009, AJ, 137, 367

Ossenkopf, V., Röllig, M., Neufeld, D. A., et al. 2013, A\&A, 550, A57

Pabst, C. H. M., Goicoechea, J. R., Teyssier, D., et al. 2017, A\&A, 606, A29

Pabst, C. H. M., Higgins, R. D., Goicoechea, J. R., et al. 2019, Nature, DOI: $10.1038 / \mathrm{s} 41586-018-0844-1$

Parikka, A., Habart, E., Bernard-Salas, J., et al. 2017, A\&A, 599, A20

Pellegrini, E. W., Baldwin, J. A., Ferland, G. J., Shaw, G., \& Heathcote, S. 2009, ApJ, 693, 285

Peng, T.-C., Wyrowski, F., Zapata, L. A., Güsten, R., \& Menten, K. M. 2012, A\&A, 538, A12

Pety, J., Guzmán, V. V., Orkisz, J. H., et al. 2017, A\&A, 599, A98

Pilbratt, G. L., Riedinger, J. R., Passvogel, T., et al. 2010, A\&A, 518, L1

Pilleri, P., Fuente, A., Gerin, M., et al. 2014, A\&A, 561, A69

Pineau des Forets, G., Flower, D. R., Hartquist, T. W., \& Dalgarno, A. 1986, MNRAS, 220, 801

Pineda, J. L., Langer, W. D., Velusamy, T., \& Goldsmith, P. F. 2013, A\&A, 554 A103

Poglitsch, A., Waelkens, C., Geis, N., et al. 2010, A\&A, 518, L2

Rahner, D., Pellegrini, E. W., Glover, S. C. O., \& Klessen, R. S. 2017, MNRAS, 470, 4453

Rodríguez-Franco, A., Martin-Pintado, J., \& Fuente, A. 1998, A\&A, 329, 1097

Rodríguez-Franco, A., Wilson, T. L., Martín-Pintado, J., \& Fuente, A. 2001, ApJ, 559,985

Rosenthal, D., Bertoldi, F., \& Drapatz, S. 2000, A\&A, 356, 705

Salas, P., Galaz, G., Salter, D., et al. 2014, ApJ, 797, 134

Schmid-Burgk, J., Densing, R., Krugel, E., et al. 1989, A\&A, 215, 150

Simón-Díaz, S., Herrero, A., Esteban, C., \& Najarro, F. 2006, A\&A, 448, 351

Stacey, G. J., Jaffe, D. T., Geis, N., et al. 1993, ApJ, 404, 219

Sternberg, A., \& Dalgarno, A. 1995, ApJS, 99, 565

Störzer, H., \& Hollenbach, D. 1998, ApJ, 495, 853

Sturm, E., Verma, A., Graciá-Carpio, J., et al. 2010, A\&A, 518, L36

Tahani, K., Plume, R., Bergin, E. A., et al. 2016, ApJ, 832, 12

Tan, J. C., Beltrán, M. T., Caselli, P., et al. 2014, in Protostars and Planets VI, eds. H. Beuther, et al. (Tucson, AZ: University of Arizona Press), 149

Tercero, B., Cernicharo, J., Pardo, J. R., \& Goicoechea, J. R. 2010, A\&A, 517, A96

Ungerechts, H., Bergin, E. A., Goldsmith, P. F., et al. 1997, ApJ, 482, 245

Usero, A., Leroy, A. K., Walter, F., et al. 2015, AJ, 150, 115

Valdivia, V., Godard, B., Hennebelle, P., et al. 2017, A\&A, 600, A114

van der Tak, F. F. S., Ossenkopf, V., Nagy, Z., et al. 2012, A\&A, 537, L10

van der Tak, F. F. S., Chavarría, L., Herpin, F., et al. 2013, A\&A, 554, A83

van Dishoeck, E. F., Kristensen, L. E., Benz, A. O., et al. 2011, PASP, 123, 138

van Dishoeck, E. F., Herbst, E., \& Neufeld, D. A. 2013, Chem. Rev., 113, 9043

Wareing, C. J., Pittard, J. M., Wright, N. J., \& Falle, S. A. E. G. 2018, MNRAS, 475, 3598

Wilson, T. L., Muders, D., Kramer, C., \& Henkel, C. 2001, ApJ, 557, 240

Wu, R., Bron, E., Onaka, T., et al. 2018, A\&A, 618, A53

Yang, B., Stancil, P. C., Balakrishnan, N., \& Forrey, R. C. 2010, ApJ, 718, 1062

Zanchet, A., Godard, B., Bulut, N., et al. 2013, ApJ, 766, 80

Zinnecker, H., \& Yorke, H. W. 2007, ARA\&A, 45, 481

Ziurys, L. M., \& Turner, B. E. 1986, ApJ, 300, L19

Zuckerman, B. 1973, ApJ, 183, 863 


\section{Appendix A: Complementary tables}

Table A.1. Line centroid LSR velocities (in $\mathrm{km} \mathrm{s}^{-1}$ ) extracted from Gaussian line fits toward selected positions.

\begin{tabular}{lrrrrrrr}
\hline \hline $\begin{array}{l}v_{\text {LSR }}\left(\mathrm{km} \mathrm{s}^{-1}\right)^{a}: \\
\text { Position }\end{array}$ & $\begin{array}{r}\text { Line: } \\
\mathrm{CH}^{+} 1-0\end{array}$ & {$[\mathrm{C}$ II $] 158 \mu \mathrm{m}$} & $\mathrm{CO} 10-9$ & $\mathrm{CO} 2-1$ & ${ }^{13} \mathrm{CO} 2-1$ & $\mathrm{C}^{18} \mathrm{O} 2-1$ & $\mathrm{HCO}^{+} 3-2$ \\
\hline$\# 1$ & $8.3(3)$ & $8.5(1)$ & $8.8(1)$ & $9.2(1)$ & $9.3(1)$ & $9.3(1)$ & $9.3(1)$ \\
$\# 2$ & $9.2(3)$ & $8.7(1)$ & $9.3(1)$ & $9.2(1)$ & $9.3(1)$ & $9.3(1)$ & $9.3(1)$ \\
$\# 3$ & $8.9(1)$ & $9.2(1)$ & $9.2(1)$ & $9.0(1)$ & $8.4(1)$ & $8.5(1)$ & $8.6(1)$ \\
$\# 4$ & $10.6(1)$ & $10.4(1)$ & $10.7(1)$ & $10.1(1)$ & $10.2(1)$ & $10.3(1)$ & $10.3(1)$ \\
$\# 5$ & $8.7(1)$ & $8.4(1)$ & $9.2(1)$ & $9.3(1)$ & $9.4(1)$ & $9.2(1)$ & $9.0(1)$ \\
$\# 6$ & $9.8(1)$ & $9.3(1)$ & $9.4(2)$ & $9.4(1)$ & $9.2(1)$ & $9.3(1)$ & $9.4(1)$ \\
$\# 7$ & $10.8(1)$ & $10.7(1)$ & $10.9(1)$ & $10.4(1)$ & $10.6(1)$ & $10.4(1)$ & $10.6(1)$ \\
$\# 8$ & $10.9(1)$ & $10.3(1)$ & $10.5(9)$ & $9.9(1)$ & $9.7(2)$ & $9.4(2)$ & $9.3(1)$ \\
$\# 9$ & $10.2(1)$ & $9.0(1)$ & $7.8(1)$ & $9.0(1)$ & $9.8(1)$ & $9.8(1)$ & $9.6(1)$ \\
$\# 10$ & $8.3(1)$ & $8.5(1)$ & $8.8(1)$ & $8.4(1)$ & $8.5(1)$ & $8.4(1)$ & $8.2(1)$ \\
\hline Average & $10(3)$ & $9.3(9)$ & $9.6(8)$ & $9.4(6)$ & $9.4(7)$ & $9.3(6)$ & $9.3(7)$ \\
\hline
\end{tabular}

Notes. ${ }^{(a)}$ From maps convolved to the angular resolution of the $\mathrm{CH}^{+} 1-0$ line $\left(27^{\prime \prime}\right) .{ }^{(b)}$ We have not used position \#9 for the averages. Parentheses indicate the uncertainty obtained by the Gaussian fitting program. The fit uncertainty is in units of the last significant digit.

Table A.2. Linewidths (in $\mathrm{km} \mathrm{s}^{-1}$ ) extracted from Gaussian line fits toward selected positions.

\begin{tabular}{|c|c|c|c|c|c|c|c|}
\hline $\begin{array}{l}\Delta v\left(\mathrm{~km} \mathrm{~s}^{-1}\right)^{a}: \\
\text { Position }\end{array}$ & $\begin{array}{r}\text { Line: } \\
\mathrm{CH}^{+} 1-0 \\
\end{array}$ & {$[\mathrm{C} \mathrm{II}] 158 \mu \mathrm{m}$} & CO 10-9 & CO 2-1 & ${ }^{13} \mathrm{CO} 2-1$ & $\mathrm{C}^{18} \mathrm{O} 2-1$ & $\mathrm{HCO}^{+} 3-2$ \\
\hline$\# 1$ & $4.9(7)$ & $4.4(1)$ & $2.3(1)$ & $3.9(1)$ & $2.6(1)$ & $2.2(1)$ & $2.7(1)$ \\
\hline \#2 & $4.4(6)$ & $4.1(2)$ & $1.8(1)$ & $4.0(1)$ & $2.5(1)$ & $2.0(1)$ & $2.5(1)$ \\
\hline \#3 & $5.7(1)$ & $4.9(1)$ & $4.4(1)$ & $4.9(1)$ & $3.8(1)$ & $3.2(2)$ & $3.8(1)$ \\
\hline \#4 & $5.3(1)$ & $4.0(1)$ & $3.4(1)$ & $4.1(1)$ & $2.6(1)$ & 2.1(1) & $3.2(1)$ \\
\hline$\# 5$ & $4.8(2)$ & $3.6(1)$ & 2.3(1) & $4.8(1)$ & $3.8(1)$ & 4.1(1) & $3.4(1)$ \\
\hline \#6 & $4.1(1)$ & $2.9(1)$ & $2.2(1)$ & $3.3(1)$ & $2.1(1)$ & $1.8(1)$ & $2.3(1)$ \\
\hline \#7 & $4.4(1)$ & $4.2(1)$ & $3.5(1)$ & $4.6(1)$ & $3.5(1)$ & $3.2(2)$ & $3.0(1)$ \\
\hline \#8 & $6.5(1)$ & $5.5(1)$ & $4.7(2)$ & $4.6(1)$ & $3.2(1)$ & $4.4(3)$ & $5.3(2)$ \\
\hline \#9 & $6.8(2)$ & $3.6(1)$ & $38.6(1)$ & $24.0(4)$ & $6.3(1)$ & $3.5(1)$ & $6.4(1)$ \\
\hline$\# 10$ & $4.4(2)$ & $3.4(1)$ & $3.5(1)$ & $5.0(1)$ & $3.3(1)$ & $3.3(1)$ & $3.5(1)$ \\
\hline Average $^{b}$ & $4.9(7)$ & $4.1(7)$ & $3.0(1)$ & $4.4(5)$ & $3.0(6)$ & $2.9(9)$ & $3.3(8)$ \\
\hline
\end{tabular}

Notes. ${ }^{(a)}$ From maps convolved to the angular resolution of the $\mathrm{CH}^{+} 1-0$ line $\left(27^{\prime \prime}\right) .{ }^{(b)}$ We have not used position \#9 for the averages. Parentheses indicate the uncertainty obtained by the Gaussian fitting program. The fit uncertainty is in units of the last significant digit.

Table A.3. Integrated-line intensities (in main brightness temperature, $\mathrm{K} \mathrm{km} \mathrm{s}^{-1}$ ) extracted from Gaussian line fits toward selected positions.

\begin{tabular}{lrrrrrrr}
\hline \hline $\begin{array}{l}W\left(\mathrm{~K} \mathrm{~km} \mathrm{~s}^{-1}\right)^{a}: \\
\text { Position }\end{array}$ & $\begin{array}{l}\text { Line: } \\
\mathrm{CH}^{+} 1-0\end{array}$ & {$[\mathrm{C} \mathrm{II}] 158 \mu \mathrm{m}$} & $\mathrm{CO} \mathrm{10-9}$ & $\mathrm{CO} 2-1$ & ${ }^{13} \mathrm{CO} 2-1$ & $\mathrm{C}^{18} \mathrm{O} 2-1$ & $\mathrm{HCO}^{+} 3-2$ \\
\hline$\# 1$ & $1.7(2)$ & $332.1(4)$ & $57.0(2)$ & $209.8(1)$ & $64.7(1)$ & $8.2(1)$ & $11.3(3)$ \\
$\# 2$ & $2.2(3)$ & $349(12)$ & $53.5(2)$ & $216.3(1)$ & $67.1(2)$ & $10.7(1)$ & $22.5(3)$ \\
$\# 3$ & $47.2(1)$ & $1048(3)$ & $408(1)$ & $446(3)$ & $111.0(1)$ & $17.6(1)$ & $75.5(2)$ \\
$\# 4$ & $26.5(1)$ & $1081(2)$ & $324.6(3)$ & $384.0(7)$ & $56.5(1)$ & $5(1)$ & $27.6(2)$ \\
$\# 5$ & $3.8(1)$ & $392(4)$ & $53.5(2)$ & $268.3(1)$ & $72.6(1)$ & $9.8(1)$ & $10.0(2)$ \\
$\# 6$ & $7.1(1)$ & $492(1)$ & $153.0(2)$ & $255.6(2)$ & $57.6(5)$ & $5.5(1)$ & $18.8(3)$ \\
$\# 7$ & $24.8(1)$ & $810(17)$ & $255.5(4)$ & $363(2)$ & $40.0(2)$ & $2.6(1)$ & $16.9(3)$ \\
$\# 8$ & $6.7(1)$ & $508(8)$ & $10.5(4)$ & $175.3(1)$ & $17.6(1)$ & $1.6(1)$ & $7.9(3)$ \\
$\# 9$ & $25.2(4)$ & $752(5)$ & 3626.3 & $1920(20)$ & $181(3)$ & $18.5(3)$ & $102(1)$ \\
$\# 10$ & $4.0(1)$ & $290(1)$ & $87.1(5)$ & $265.8(2)$ & $52.8(1)$ & $6.8(1)$ & $20.5(3)$ \\
\hline Average $^{b}$ & $14(15)$ & $589(292)$ & $156(133)$ & $287(85)$ & $60(24)$ & $8(5)$ & $23(19)$ \\
\hline
\end{tabular}

Notes. ${ }^{(a)}$ From maps convolved to the angular resolution of the $\mathrm{CH}^{+} 1-0$ line $\left(27^{\prime \prime}\right) .{ }^{(b)}$ We have not used position \#9 for the averages. Parentheses indicate the uncertainty obtained by the Gaussian fitting program. The fit uncertainty is in units of the last significant digit. 


\section{Appendix B: Complementary figures}
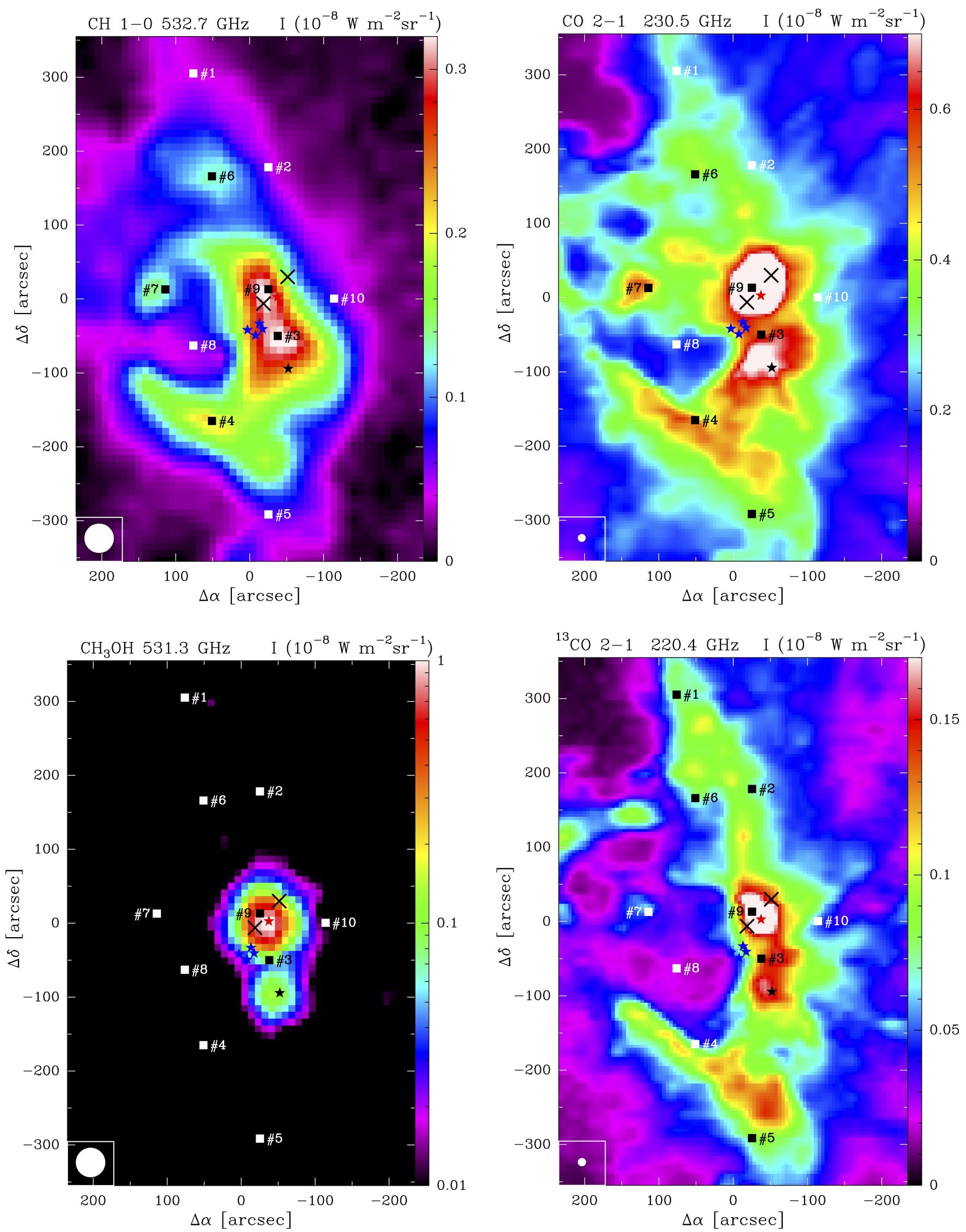

Fig. B.1. Herschel/HIFI and IRAM 30m maps of different molecular emission lines toward OMC-1. The color scale shows the integrated line intensity in $\mathrm{W} \mathrm{m}^{-2} \mathrm{sr}^{-1}$. The native angular resolution of each observation, the HPBW, is plotted in the bottom-left corner. Representative positions discussed in the text are indicated with numbers (see Sect. 4.1). 
J. R. Goicoechea et al.: Stellar radiative feedback in OMC-1
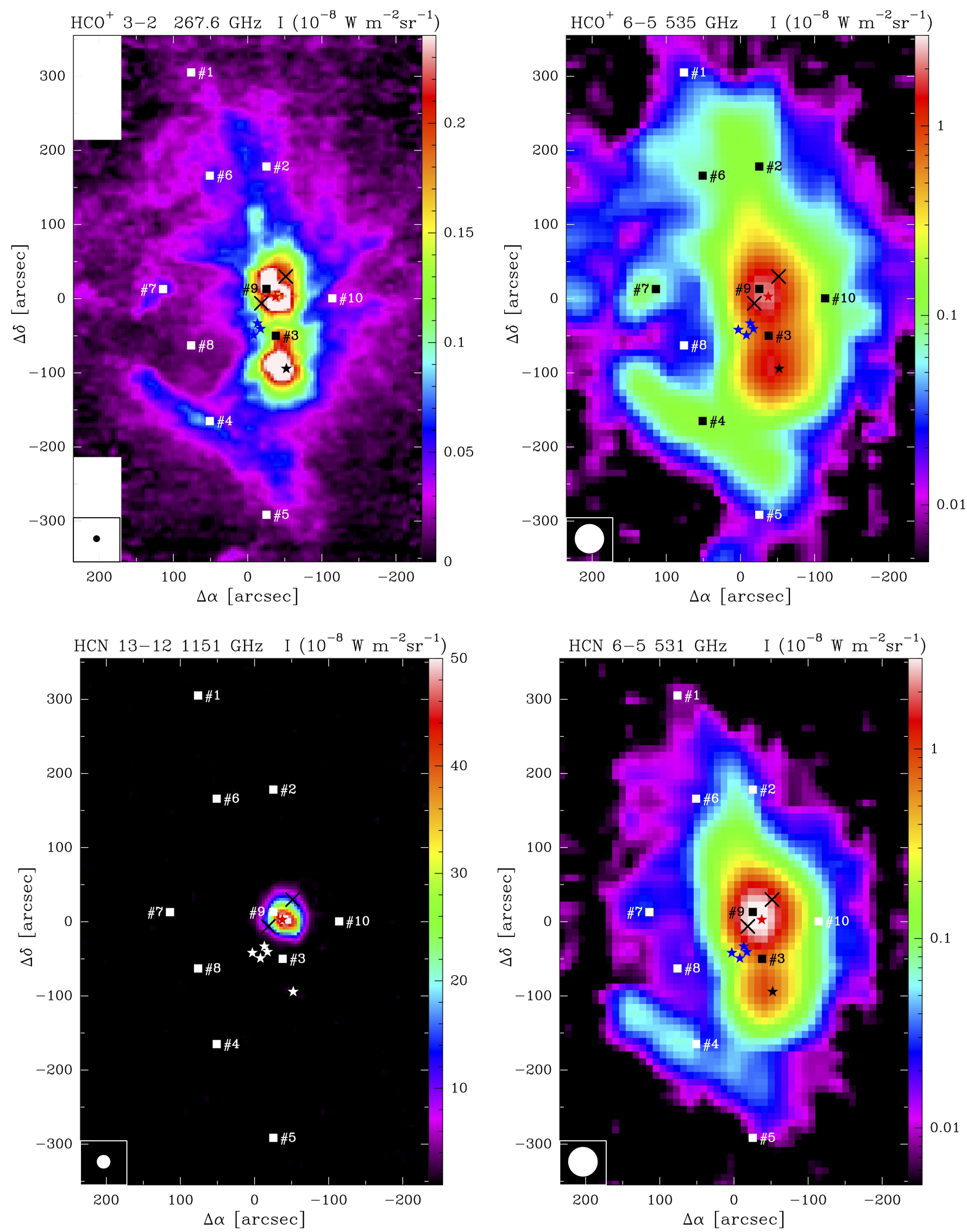

Fig. B.2. Herschel/HIFI and IRAM $30 \mathrm{~m}$ maps of different high critical-density tracers (mid- $J$ HCN and $\mathrm{HCO}^{+}$lines). The color scale shows the integrated line intensity in $\mathrm{W} \mathrm{m}^{-2} \mathrm{sr}^{-1}$. The native angular resolution of each observation, the HPBW, is plotted in the bottom-left corner. Representative positions discussed in the text are indicated with numbers (see Sect. 4.1). 
A\&A 622, A91 (2019)
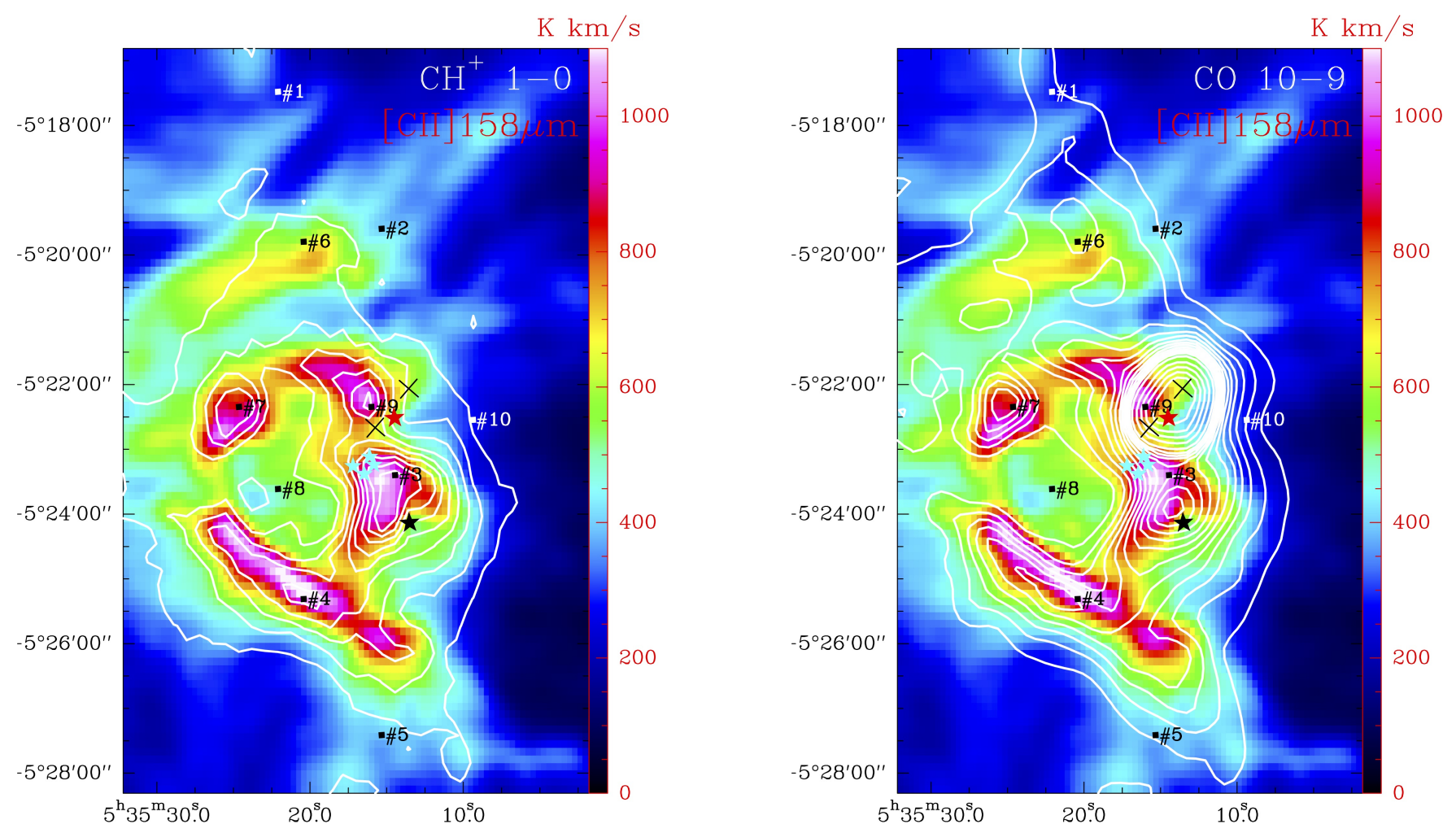

Fig. B.3. Integrated line emission from [C II $158 \mu \mathrm{m}$ (in color scale; Goicoechea et al. 2015a), $\mathrm{CH}^{+} J=1-0$, and CO $J=10-9$ in white contours. Contours go from 5 to $45 \mathrm{~K} \mathrm{~km} \mathrm{~s}^{-1}$ in steps of $5 \mathrm{~K} \mathrm{~km} \mathrm{~s}^{-1}$ for $\mathrm{CH}^{+}$, and from 50 to $600 \mathrm{~K} \mathrm{~km} \mathrm{~s}^{-1}$ in steps of $50 \mathrm{~K} \mathrm{~km} \mathrm{~s}^{-1}$ and $700-1600 \mathrm{~K} \mathrm{~km} \mathrm{~s}^{-1}$ in steps of $200 \mathrm{~K} \mathrm{~km} \mathrm{~s}^{-1}$ for CO. Maps have been convolved to the same angular resolution (27").
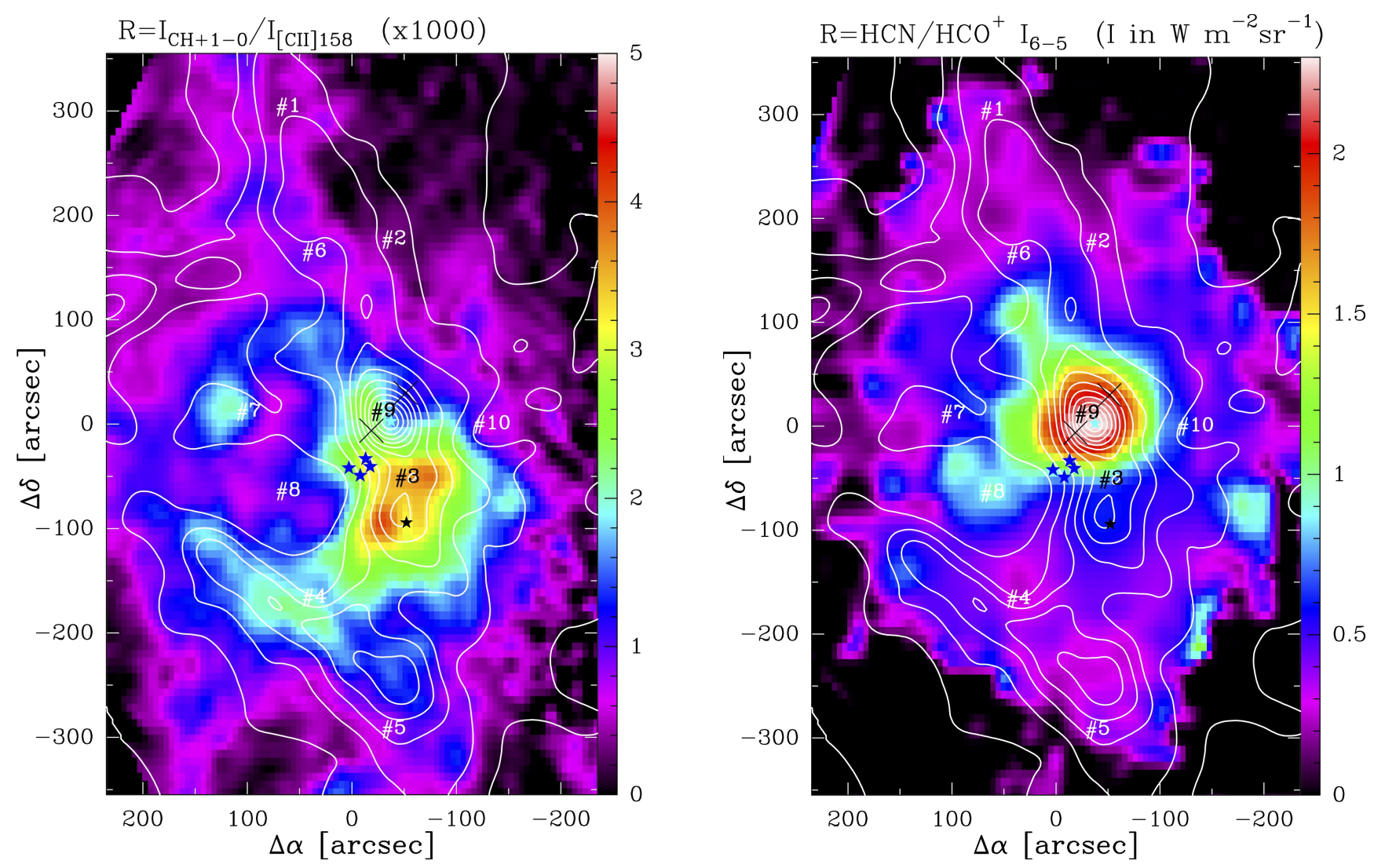

Fig. B.4. Left panel: map of the $\mathrm{CH}^{+} J=1-0$ to [C $\left.\mathrm{II}\right] 158 \mu \mathrm{m}$ line intensity ratio (in units of $\mathrm{W} \mathrm{m}^{-2} \mathrm{sr}^{-1}$ ) roughly proportional to gas density variations at the irradiated cloud surfaces. Right panel: map of the $\mathrm{HCN} J=6-5$ to $\mathrm{HCO}^{+} J=6-5$ line intensity ratio. In both maps contours represent the ${ }^{13} \mathrm{CO} J=2-1$ optically thin emission. 
J. R. Goicoechea et al.: Stellar radiative feedback in OMC-1
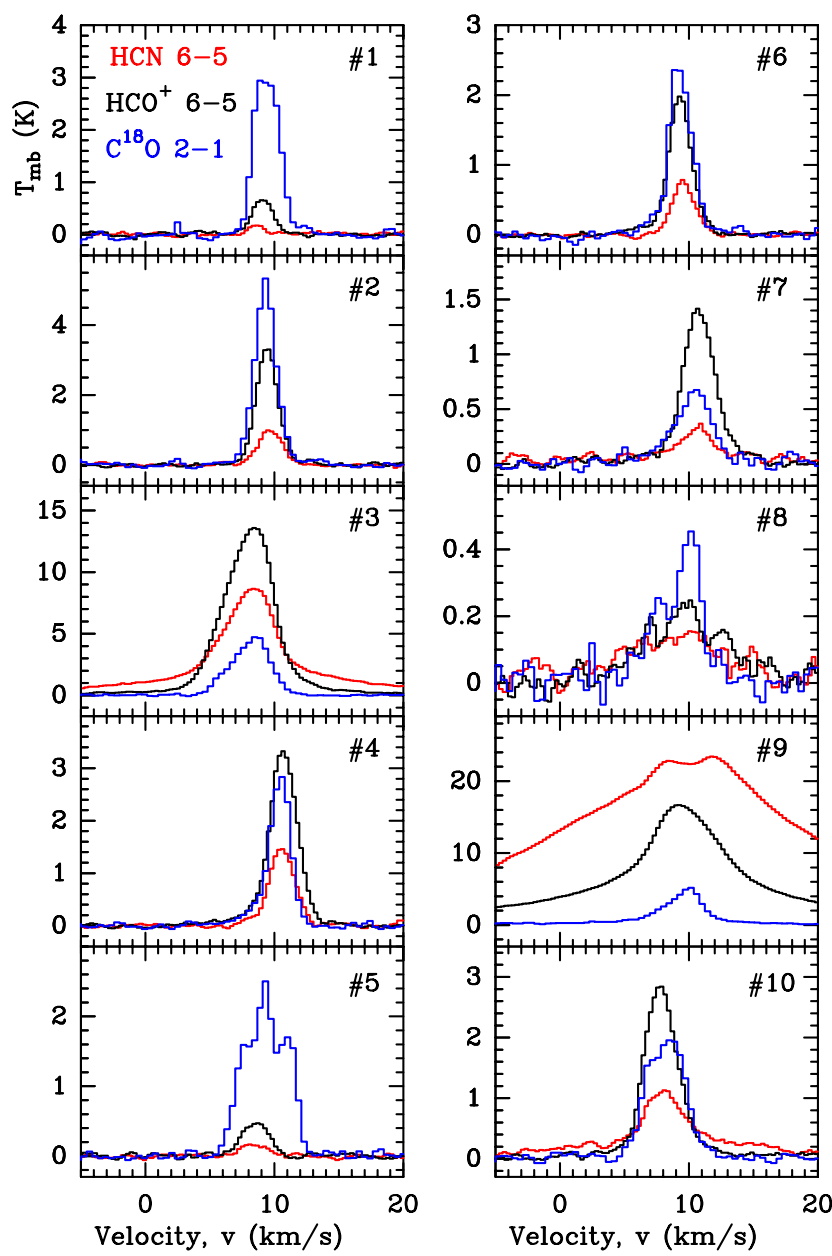

Fig. B.5. Line profiles at representative positions (see Table 3). Spectra were extracted from maps convolved to a uniform resolution of $43^{\prime \prime}$.

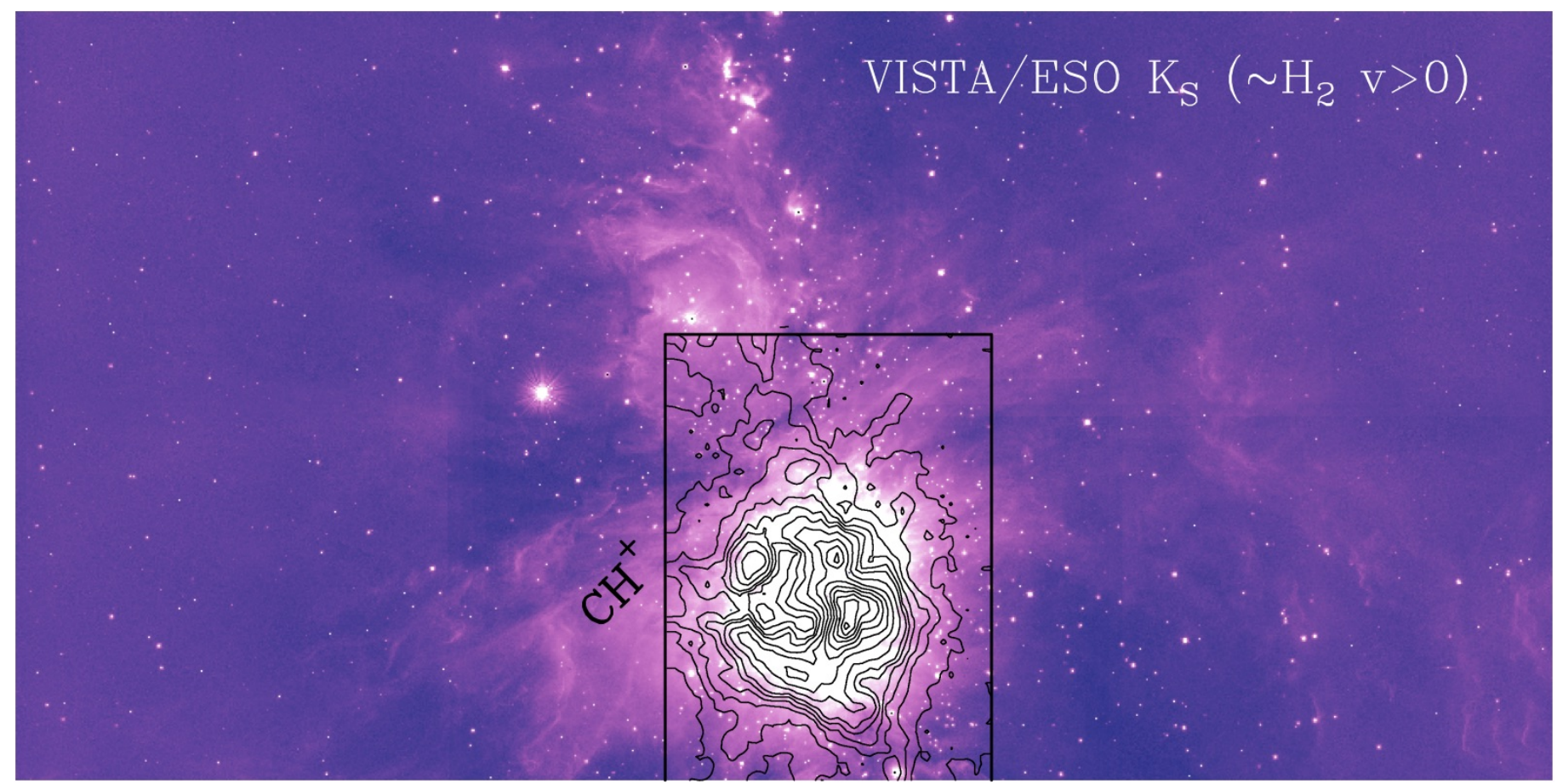

Fig. B.6. Large-scale $\left(40^{\prime} \times 20^{\prime}\right)$ VISTA-NIR image of M42 and M43 in the $K_{S}$ band around $2.15 \mu$ m (Meingast et al. 2016) tracing vibrationally excited $\mathrm{H}_{2}$ emission. The intensity scale is chosen to enhance the faint and extended NIR emission. Black contours show the $\mathrm{CH}^{+} J=1-0$ line integrated intensities from 1 to $50 \mathrm{~K} \mathrm{~km} \mathrm{~s}^{-1}$. 


\section{Appendix C: Warm gas mass from CO $J=10-9$}

In Sect. 4.2 we estimated the mass contained in the high-pressure molecular PDR layers, $M_{\mathrm{mPDR}}\left(\mathrm{H}_{2}\right)$. In particular, we integrate the column density for optically thin line emission:

$N_{\text {thin }}(\mathrm{CO})=8 \pi \frac{v^{3}}{c^{3}} \frac{Q\left(T_{\text {rot }}\right)}{g_{10} A_{10-9}} \frac{1}{1-e^{E_{u} / k T_{\text {rot }}}} \frac{W_{10-9}}{J\left(T_{\text {rot }}\right)-J\left(T_{\mathrm{bg}}\right)}\left(\mathrm{cm}^{-2}\right)$

over the OMC-1 map. $W_{10-9}$ refers to the CO $J=10-9$ line integrated intensity in $\mathrm{K} \mathrm{km} \mathrm{s}^{-1}$. We assume that the rotational temperature at each position of the map, $T_{\text {rot }}$, is the same as $T_{\text {rot }}(2-1)$. Because the CO $J=2-1$ line emission is optically thick through most of the field, we can determine $T_{\text {rot }}$ at each pixel of the map from the line peak temperature, $T_{\text {peak }}(2-1)$ (see Fig. C.1), using

$$
J\left(T_{\text {rot }}\right)=T_{\text {peak }}(2-1)+J\left(T_{\text {bg }}\right),
$$

where $J(T)=T^{*} /\left(e^{T^{*} / T}-1\right)$ is the equivalent brightness temperature, $T^{*}=E_{\mathrm{u}} / k$, and $T_{\mathrm{bg}}$ is the temperature of the cosmic microwave background. If the CO $J=10-9$ emission is slightly optically thick, line opacity $\tau_{10-9} \simeq 1$, we add an optical depth correction $N(\mathrm{CO})=N_{\text {thin }}(\mathrm{CO}) \cdot \tau_{10-9} /\left(1-e^{-\tau_{10-9}}\right)$, where

$\tau_{10-9}=-\ln \left[1-\frac{T_{\mathrm{P}}(10-9)}{J\left(T_{\mathrm{rot}}\right)-J\left(T_{\mathrm{bg}}\right)}\right]$,

is calculated from observations. Once we created a map of the warm CO column density from the CO $J=10-9$ emission, we convert it to a total gas column density map through $N_{\mathrm{H}}=x(\mathrm{CO}) \cdot N(\mathrm{CO}) \simeq 10^{-4} \cdot N(\mathrm{CO})$ using a representative value for the $\mathrm{CO}$ abundance in the PDR. The mass of the warm PDR molecular gas in OMC- 1 is then $M(\mathrm{mPDR}) \simeq \mu m_{\mathrm{H}} \sum_{i}\left(N_{\mathrm{H}}^{i} A_{i}\right)$, where $A_{i}$ is the pixel $i$ area, $m_{\mathrm{H}}$ is the $\mathrm{H}$ atom mass, and $\mu$ is the mean molecular weight. For this mass estimation, we used the CO $J=2-1$ and 10-9 maps convolved to $27^{\prime \prime}$.

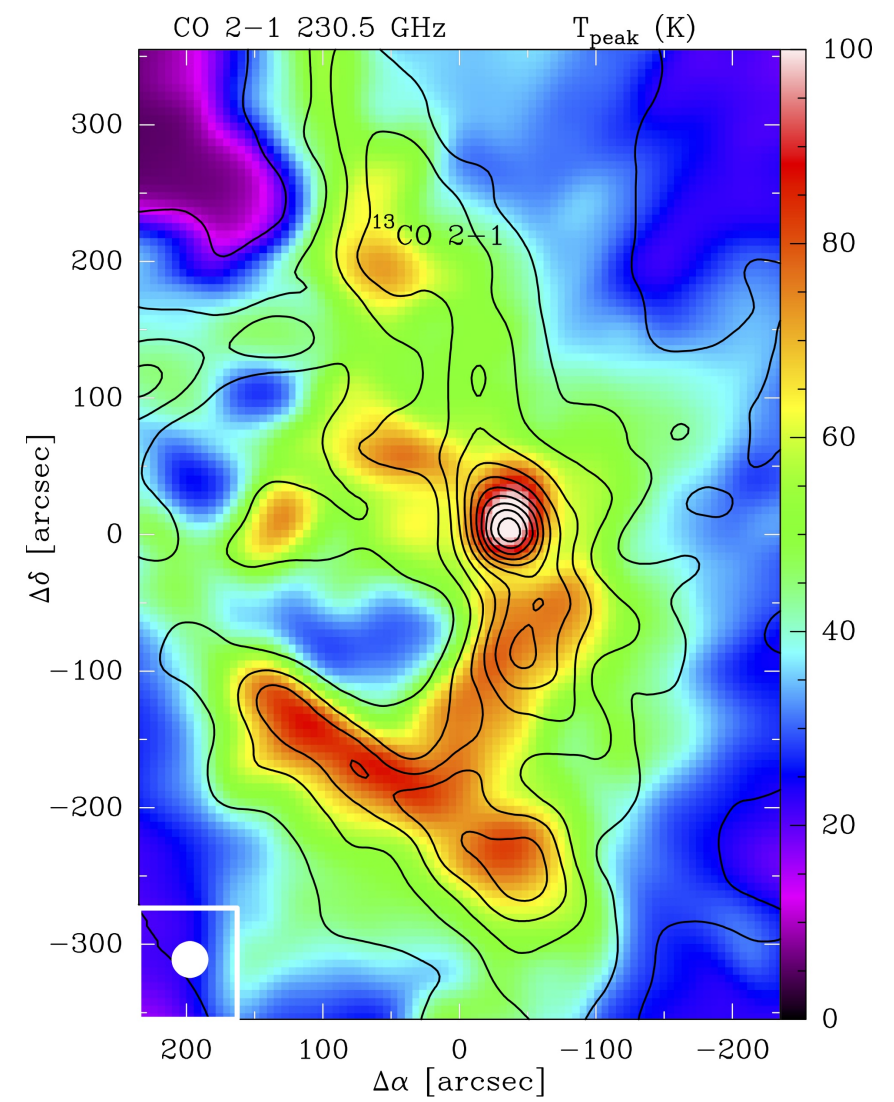

Fig. C.1. Map of the CO $J=2-1$ line intensity peak ( $T_{\text {peak }}$ in $\mathrm{K}$; shown in color scale). Because the CO $J=2-1$ emission is optically thick in most of the field, this map provides a lower limit to the gas temperature in the layers where the line emission arises, that is, $T_{\text {peak }} \simeq T_{\text {rot }}$ $(\mathrm{CO} 2-1) \leq T_{\mathrm{k}}$. 